NOTICE WARNING CONCERNING COPYRIGHT RESTRICTIONS:

The copyright law of the United States (title 17, U.S. Code) governs the making of photocopies or other reproductions of copyrighted material. Any copying of this document without permission of its author may be prohibited by law. 


\section{NETWORKS OF CONSTRAINTS: FUNDAMENTAL}

PROPERTIES AND APPLICATIONS TO

PICTURE PROCESSING

by

Ugo Montanari

January, 1971

Department of Computer Science Carnegie-Mellon University Pittsburgh, Pennsylvania 


\section{Networks of constraints: fundamental properties and applications to picture processing *}

by Ugo Montanari

\section{Abstract}

The problem of representation and handling of constraints is here considered, mainly for picture processing purposes. A systematic specification and utilization of the avallable constraints could significantly reduce the amount of search in picture recognition. On the other hand, formally stated constraints can be embedded in the syntactic productions of picture languages. Only binary constraints are treated here, but they are represented in full generality as binary relations. Constraints among more than two variables are then represented as networks of simultaneous binary relations. In general, more than one equivalent (i.e., representing the same constraint) network can be found: a minimal equivalent network is shown to exist, and its computation is shown to solve most practical problems about constraint handing. No exact solution for this central problem was found. Anyway, constraints are treated algebraically, and the solution of a system of linear equations in this algebra provides an approximation of the minimal network. This solution is then proved exact in special cases, e.g., for tree-like and series parallel networks and for classes of relations for which a distributive property holds. This latter condition is satisfied in cases of practical interest.

\footnotetext{
"This work was supported by the Advanced Research Projects Agency of the office of the Secretary of Defense (F44620-70-C-0107) and is monitored by the Air Force Office of Scientific Research. This document has been approved for public release and sale; its distribution is unlimited.
} 


\section{Introduction}

In writing this paper we had in mind mainly the problems of a particular field, namely picture recognition and description. However, the problem of proper representation and economic handing of constraints is very general and is important in many problems of operations research, engineering and computer science. For instance, many practical design problems consist of finding any solution which satisfies all topological and geometrical restrictions [1]. Even when an optimization problem must be stated, the chosen constraint representation is essential in determining the nature of the mathematical problem involved and its difficulty. Unfortunately, many practical constraints are difficult to handle, because they involve in a complicated way many variables. For instance, we can mention the noncrossing condition among electrical paths in an integrated circuit layout or as a more esoteric example, the restriction to be faced in the design of computer rooms that all magnetic tape units must be in sight from the operator.

In picture processing, constraints play an important role, but they are unlikely to be representable in a linear or anyway simple form. Here constraints are better known by the name of (geometrical, topological, structural) properties of the class of picture under consideration. But in fact they are present as fixed characteristics in explicit models or are implied by recognition routines which do not take into account configurations without the desired property.

However, we believe that an explicit and consistent treatment of constraints can bring valuable advantages. To show what we have in mind, we present some scenarios. 
a) Speed up of the recognition process. Often recognition subroutines search a picture for specific elements or features. The search space is usually more than two-dimensional, because other free parameters must be determined at the same time (e.g., the angular position of a stroke, the vertex structure in a cube [2]). For efficiency, what we are looking for must in general be dependent on what we have already found out about the particular picture. More precisely, it is useless to look for features which are possible a priori, but are not consistent with the part of the picture we have already recognized. For instance, if the problem is to recognize human faces [3] we must of course limit the search for particular elements (eyes, nose, mouth, ears, etc.) to the areas of the picture where they may ever be present. A second step is to establish constraints between pairs of elements. If for instance the position of one ear has already been determined, the area in which the mouth could be found is further restricted. Such binary constraints, if formally stated, can be intersected and composed. For instance, if also an eye has been determined, the allowed area for the mouth can be considered the intersection of the constraints given by the ear and the eye. Furthermore, the presence of elements yet unfound, such as the nose, but for which constraints have been defined, could transmit further constraints from determined elements to the sough ones. In conclusion, if all those constraints are superimposed, the search space can be reduced. Only the first few elements will be time consuming. For the others, the recognition procedure should be essentially a check of the evidence we have already gathered.

b) Optimal recognition. In particularly bad cases, combined evidence from all'elements is required before accepting a picture, because many acceptable 


$$
=4=
$$

candidates are present for each feature. This situation arises mainly during preprocessing or anyway during recognition of unstructured entities (see [4] for the limit case of optimal detection of curves). In this case it is convenient to assign a merit figure to the various alternatives, and then to find the best one with an optimization procedure. Again, systematic handling of constraints is vital in reducing the combinatorics involved.

c) Imperfect models. A model can be imprecise because it is too simple, or because something present in the model is missing in reality. In the former case the model will not be as powerful as it could be, but it will work; while in the latter case the picture could be rejected as not satisfying the model. The missing part could be simply obscured by the noise. If the model is organized in terms of constraints, a model without the critical part could be systematically built taking into account the constraints transmitted from one part of the model to the other through the missing part.

d) Linguistic methods. The application of parsing mechanisms in picture analysis is very promising $[5,6]$. These methods work well if the structure of the image is mainly topological, as in bubble chamber tracks, chemical structures, block diagrams [7,8]. If geometrical information is essential, it can be embedded in the syntax rules only in simple cases, as in the linguistic description of mathematical formulas [9]. If the allowed geometrical relations can be expressed as a set of simultaneous constraints, they can be formally added to the rewriting rules of the grammar under the form of applicability conditions [10]. In this way, perhaps the geometrical consistency of the various rules can be proved at a grammar level. This fact would guarantee that all the pictures generated by the grammar are consistent and representable on the plane. 


$$
=5=
$$

In this paper we have limited our formalization to binary constraints. On the other hand, they are represented in the most general way, i.e., as algebraic relations between sets of possible values of pair of variables [14]. For many variables, a constraint is then represented as a network of simultaneous binary relations.

Of course, an n-ary constraint cannot always be represented exactly by an n-vertex network of binary constraints. However an optimal approximating network can be given easily. On the other hand, many different but equivalent networks can represent the same n-ary constraint. A11 the networks equivalent to a given one can be ordered by set inclusion. A least element is proved to exist and it is called the minimal network. Minimal networks are shown to have all the constraints as explicit as possible.

The problem of the determination of the minimal network from a given one is then shown to include most of the practical problems mentioned above about constraint composition and transmission. Unfortunately, no general algorithm was found. This is not surprising, since very tough problems, like the graph coloring problems, fit this scheme.

Approximate solutions are considered instead. In those networks (which are called closed) all those global constraints are explicit that can be transmitted through all the possible paths in the network. The problem of determining a closed, equivalent network is then stated algebraically. If the operations of intersection and composition of constraints are defined, the above problem can be shown equivalent to the solution of a system of linear equations in this algebra. Since composition does not distribute, in general, over intersection, an iterated Gaussian elimination algorithm is required for finding the solution of the system. 


$$
=6=
$$

In the last section, some special cases are investigated, in which closed networks are minimal, i.e., in which our algorithm computes the exact solution. If the topology of the network is restricted, tree-like and series-parallel closed networks are proved minimal. The same result is also achieved if we restrict the class of allowed relations to a class where composition distributes over intersection. This is the case when the sets of possible values of variables have a lattice structure and the relations satisfy a monotonicity assumption. This condition is satisfied in some cases of practical interest, such as the shortest path problem in a graph (possibly with negative weights) and its multimensional equivalents. Here our algorithm becomes the well-known Floyd algorithm [13]. 


\section{Union, intersection_and composition of constraints}

In this section a constraint between two variables is represented, in complete generality, by a relation between two sets. Elementary algebraic properties of relations are then recalled.

If a constraint exists between two variables $x_{1}$ and $x_{2}, x_{1} \in x_{1}=\left\{x_{1}, \ldots, x_{1}, N_{1}\right\}$,

$x_{2} \in x_{2}=\left\{x_{2}, 1, \ldots, x_{2, N_{2}}\right\}$ then in general not all possible pairs $\left(x_{1, r}, x_{2, s}\right)$ are allowed. The set of allowed pairs is called a relation between sets $X_{1}$ and $X_{2}$. In general, it is convenient to consider ordered pairs and thus to distinguish between a relation $R_{12}$ and a relation $R_{21}$. For instance, if $x_{1}=\{1,2\}$ and $x_{2}=\{1,2,3\}$ then $R_{12}=\{(1,1),(2,1),(1,3)\}$ is a relation. Any relation $R_{12}$ is thus a subset, proper or improper, of the product set $X=X_{1} x_{2}$ of all the pairs. A standard way of representing subsets is to use the characteristic function $\mathrm{F}$ :

$$
F: X_{1} \times X_{2} \rightarrow\{0,1\} \quad ; \quad F\left(\left(x_{1, r}, x_{2, s}\right)\right)=1 \text { iff }\left(x_{1, r}, x_{2, s}\right) \in R_{12}
$$

In other words to each pair in $X$ a binary digit is associated, which is 1 if and only if the pair belongs to the relation. Being characterized by a binary number of $\mathrm{N}_{1} \cdot \mathrm{N}_{2}$ digits, $2^{\mathrm{N}_{1} \cdot \mathrm{N}_{2}}$ different relations exist between $X_{1}$ and $\mathrm{X}_{2}$. Usually, these digits are arranged in a $\mathrm{N}_{1} \times \mathrm{N}_{2}$ matrix $\left[\mathrm{R}_{12, \mathrm{rs}}\right]$ whose rows correspond to the elements of set $X_{1}$ and columns to set $X_{2}$. Thus we have:

$$
R_{12, r s}=1 \text { iff }\left(x_{1, r}, x_{2, s}\right) \in R_{12}
$$

In our example, the characteristic binary matrix is:

$$
R_{12}=\left|\begin{array}{lll}
1 & 0 & 1 \\
1 & 0 & 0
\end{array}\right|
$$

In what follows, relations will be mainly represented in natrix notation. The inverse $R_{12}^{-1}$ of a relation $R_{12}$ is defined as the transpose: $\mathrm{R}_{12, \mathrm{rs}}^{-1}=\mathrm{R}_{12, \mathrm{rs}}^{\mathrm{T}}=\mathrm{R}_{21, \mathrm{sr}}$ 
For relations, being sets, we can define the usual operations of negation

$$
\bar{R}_{12}=7 R_{12} \text { iff } \quad \bar{R}_{12, \mathrm{rs}}=7 R_{12, \mathrm{rs}} \quad\left(r=1, \ldots, N_{1} ; s=1, \ldots, N_{2}\right)
$$

union or logical sum

$$
R_{12}=R_{12}^{\prime} \cup R_{12}^{\prime \prime} \text { iff } R_{12, r s}=R_{12, r s}^{i} V R_{12, r s}^{\prime \prime}
$$

intersection or logical product

$$
R_{12}=R_{12} \cap R_{12}^{\prime \prime} \quad \text { iff } \quad R_{12, r s}=R_{12, r s}^{\prime} \wedge R_{12, r s}^{\prime \prime}
$$

and the partial ordering relation of set inclusion

$$
\mathrm{R}_{12}^{\prime} \subseteq \mathrm{R}_{12}^{\prime \prime} \text { j.ff } \quad \mathrm{R}_{12, \mathrm{rs}}^{\prime} \subset \mathrm{R}_{12, \mathrm{rs}}^{\prime \prime}
$$

An empty relation $\phi_{12}$ and an universal relation $U_{12}$ can be defined

$$
\sigma_{12, \mathrm{rs}}=0 \quad ; \quad \mathrm{U}_{12, \mathrm{rs}}=I
$$

such that

$$
R_{12} \cup \sigma_{12}=R_{12} \quad ; \quad R_{12} \cap v_{12}=R_{12}
$$

for all $R_{12}$. Thus relations between two sets form a complete lattice with greatest element $U$ and least element $\emptyset$ and where the operations of sup and inf coincide with union and intersection respectively.

Next step is to consider a constraint $R_{12}$ between variables $x_{1}$ and $x_{2}$ and a constraint $R_{23}$ between variables $x_{2}$ and $x_{3}$. There will be an induced or transmitted constraint $R_{13}$ between variables $x_{1}$ and $x_{3}:$ a pair $\left(x_{1, r}, x_{3, s}\right)$ is allowed, if at least one value $x_{2, t}$ exists, such that both $\left(x_{1, r}, x_{2, t}\right)$ and $\left(x_{2, t}, x_{3, s}\right)$ are allowed by $R_{12}$ and $R_{23}$ respectively. This requirement defines the operation of composition of relations:

$$
R_{13}=R_{12} \cdot R_{23} \text { iff } R_{13, r s}=V_{t=1}^{N} R_{12, r t} \wedge R_{23, t s}
$$

Note that composition, in matrix nctation, is just binary matrix multiplication. For example, we may have

$$
R_{12}=\left|\begin{array}{lll}
1 & 0 & 1 \\
1 & 0 & 0
\end{array}\right| ; \quad R_{23}=\left|\begin{array}{ll}
1 & 0 \\
1 & 1 \\
0 & 1
\end{array}\right| ; \quad R_{13}=R_{12} \cdot R_{23}=\left|\begin{array}{ll}
1 & 1 \\
1 & 0
\end{array}\right|
$$


It is very easy to see that composition is associative, and that an identity relation exists, defined only between a set and itself,

$$
I_{I l, r s}=1 \text { iff } r=s
$$

such that

$$
R_{12} \cdot I_{22}=I_{11} \cdot R_{12}=R_{12}
$$

for every relation $R_{12}$.

The defined operations of union, intersection and composition have an useful monotoniclty property. If $f\left(R_{12}\right)$ is any expression involving the operations of intersection, union and composition among relation $R_{12}$ and any number of constants, from $R_{12}^{\prime} \subseteq R_{12}^{\prime \prime}$ we have $f\left(R_{12}^{\prime}\right) \subseteq f\left(R_{12}^{\prime \prime}\right)$. This property is obvious if we notice that function $f$, written in binary form, contains binary sums and products, but no negetions.

A particular case of a relation happens when one of the two sets (say the first) has just one element. These relations, in binary form, are representable as vectors and are in a one-to-one correspondence with the subsets of the second set. Actually, in what follows we will always assume the existence of a fictitious one-element set $x_{0}$, to have an homogeneous way of representing subsets. Especially useful in our formalism are the fundamental vectors $\mathrm{V}_{01}$ and $\mathrm{V}_{10}$, 1.e., the vectors with only one nonzero element. For instance, the image in $\mathrm{R}_{12}$ of the element $x_{1, r}$ can be defined as represented by the vector: $\mathrm{R}_{02}=\mathrm{V}_{\mathrm{O} 1} \cdot \mathrm{R}_{12}$

where $V_{O I}$ is the fundamental vector corresponding to element $x_{1, r}$ : $V_{01, t}=1$ iff $t=r$

A relation $R_{12}$ is called total if every element of $x_{1}$ and $x_{2}$ is in relation with some other element. In our formalism, $R_{12}$ is total iff 


$$
=10=
$$

$v_{01} \cdot R_{12} \neq \phi_{02}$ and $R_{12} \cdot v_{20} \neq \phi_{10}$

for every fundamental vector $V_{01}$ and $V_{20}$. Given any total relation $R_{12}$, it is easy to see that $R_{12} \cdot U_{23}=U_{13}$ and $U_{11} \cdot R_{12}=U_{12} \cdot$

In what follows we are mainly interested in the operations of intersecttron and composition, so we will use the symbol + for intersection and the simple concatenation for composition. Unfortunately, composition does not distribute over intersection. In general

$$
R_{12}\left(R_{23}^{\prime}+R_{23}^{\prime \prime}\right) \neq R_{12} R_{23}^{\prime}+R_{12} R_{23}^{\prime \prime}
$$

For instance, if

$$
R_{12}=\left|\begin{array}{ll}
1 & 1 \\
0 & 0
\end{array}\right| ; \quad R_{23}^{\prime}=\left|\begin{array}{ll}
0 & 0 \\
1 & 0
\end{array}\right| ; \quad R_{23}^{\prime \prime}=\left|\begin{array}{ll}
1 & 0 \\
0 & 0
\end{array}\right|
$$

then

$$
\mathrm{R}_{12}\left(\mathrm{R}_{23}^{\prime}+\mathrm{R}_{23}^{\prime \prime}\right)=\emptyset_{13} \quad ; \quad \mathrm{R}_{12} \mathrm{R}_{23}^{\prime}+\mathrm{R}_{12} \mathrm{R}_{23}^{\prime \prime}=\left|\begin{array}{ll}
1 & 0 \\
0 & 0
\end{array}\right|
$$

A special case arises when distributivity does hold. In general; we say that the relations

$$
R_{i k}, R_{k i} \quad i=1, \ldots, n ; 1 \neq k
$$

form a distributive set of relations with respect to set $x_{k}$ if (2.1) $\left(\sum_{\substack{i=1 \\ i \neq k}}^{m} v_{O i} R_{i k}\right)\left(\sum_{\substack{i=1 \\ i \neq k}}^{m} R_{k 1} v_{10}\right)=\sum_{\substack{i=1 \\ i \neq k}}^{m} \sum_{\substack{j=1 \\ j \neq k}}^{m} v_{0 i} R_{1 k} R_{k j} v_{j O}$ for every set of fundamental vectors $v_{01}, v_{10}(1=1, \ldots, m ; i \neq k)$ and for every $m$. In (2.1) the indexes of the sums go from 1 to $m$. Actually, the ordering is immaterial and thus we require (2.1) to hold whenever the indexes assume any set of $\mathrm{m}$ values. Note that distributivity defined in terms of fundamental vectors is more general than simple distributivity. For instance, from

$$
v_{01} R_{12}\left(R_{23}^{\prime} v_{30}^{\prime}+R_{23}^{\prime \prime} v_{30}^{\prime \prime}\right)=v_{01} R_{12} R_{23}^{\prime} v_{30}^{\prime}+v_{01} R_{12} R_{23}^{\prime \prime} v_{30}^{\prime \prime}
$$

for every $v_{0,1}, v_{30}^{\prime}$ and $v_{30}^{n},(2.2)$ follows, but not conversely.

(2.2) $R_{12}\left(R_{23}^{\prime}+R_{23}^{\prime \prime}\right)=R_{12} R_{23}^{\prime}+R_{12} R_{23}^{\prime \prime}$ 


\section{Networks of constraints}

In this section, constraints among $n(n>2)$ variables are considered. A straightforward formalization of such constraints as n-ary relations is possible, but the quantity of information involved grows exponentially with $n$, and so no hope exists to handle it for any practical n. Networks of binary relations as defining an n-ary relation are then introduced. An optimal approximation theorem is proved, and just one minimal network is shown to exist. Finally, a problem is stated, called the central problem, which embodies most practical problems posed by networks of constraints.

Generalizing the approach followed in section 2, it is clear that an n-ary constraint can be considered to allow some (or none or all) among the possible $n$-tuples of values of $n$ variables. Thus an n-ary relation $\rho$ is any subset of $X=X_{1} \times X_{2} \times \ldots \times X_{n}$. The set $X$ can be visualized as an $n$-dimensional space. An n-ary relation $\rho$ thus represents a "solid" in this space. Given an m-dimensional subspace $s=x_{i_{l}} x \ldots x x_{i_{m}}$, any n-tuple a and any n-ary relation $\rho$ in $X$ can be projected on $S$ yelding the $m$-tuple $a_{S}$ and the $\mathrm{m}$-ary relation $\mathrm{P}_{\mathrm{S}}$. The number of distinct $\mathrm{n}$-tuples is $\mathrm{N}_{1} \ldots \mathrm{N}_{\mathrm{n}}$ and thus $2^{N_{1} \cdots N_{n}}$ is the number of distinct $n$-ary relations. If $N_{1}=\ldots=N_{n}=N$ then $\mathrm{N}^{\mathrm{n}}$ bits are required on the average for storing a $\mathrm{n}$-ary relation. Practical values for $\mathrm{N}$ and $\mathrm{n}$ in picture processing applications are 1000 and 20 , and thus the information involved is enormous. One way out is to consider a restricted class of n-ary relations.

A network $R$ of binary relations is defined as a set of sets $\bar{x}=\left\{x_{1}, \ldots, x_{n}\right\}$ plus a relation $R_{i j}$ from every set $x_{i}$ to every set $x_{j}(i, j=1, \ldots, n)$. Furthermore, $R_{i i} \subseteq I_{i i}(i=1, \ldots, n)$. If $R_{1 j}=R_{j 1}^{T}$ the network will be called symmetric. 
The network of relations $R$ can be thought of as representing an n-ary relation:

$$
\rho=\left\{a \mid a \in X=x_{1} x \ldots x x_{n} ;(\forall i, j) s=x_{1} \times x_{j} ; a_{s} \in R_{i j}\right\}
$$

In other words an $n$-tuple a is allowed by $\rho$ iff its projections on all the two-dimensional subspaces $S$ of $X$ simultaneously satisfy the binary constraints of the network $R$. Note that if some $R_{i j}=\emptyset_{i j}$ then $\rho=\phi$, while if $p=X$ then $R_{i j}=U_{i j}(i, j=1, \ldots, n ; i \neq j)$ and $R_{i i}=I_{1 i}(i=1, \ldots, n)$.

An obvious way of visualizing a network is by a directed graph. Vertices $v_{1}, \ldots, v_{n}$ correspond to sets $x_{l}, \ldots, x_{n}$, and an arc $v_{i} V_{j}$ is present from $v_{i}$ to $V_{j}$ iff $R_{i j} \neq V_{i j}(i \neq j)$ or $R_{i i} \neq I_{i i}$. Relation $R_{i j}$ is then associated with the direct arc $V_{i} V_{j}$. For instance the following $n$-ary relation $\rho$ is represented by the network $R$ in Fig. 1a:

$$
p=\left\{\left(x_{1,1}, x_{2,1}, x_{3,1}\right),\left(x_{1,1}, x_{2,1}, x_{3,2}\right),\left(x_{1,2}, x_{2,3}, x_{3,1}\right)\right\}
$$

or, in a more compact notation,

(3.1) $P=\left|\begin{array}{lll}1 & 1 & 2 \\ 1 & 1 & 3 \\ 1 & 2 & 1\end{array}\right|$

where the indexes of the allowed n-tuples form the columns.

A network $R$ is determined by giving in orderly fashion all its binary relations, and this requires roughly $B=n^{2} N^{2}$ bits. Clearly $B<N^{n}$ except for very small values of $N$ and $n$. This argument shows that the class of $n$-ary relations representable by networks is narrower (in fact, much narrower) than the class of all n-ary relations.

Given an $n$-ary relation $P$, the simple projection formula (3.2) generates a network $R^{\prime}$ which is, in a sense, the best possible excess approximation of $p$. (3.2) $R_{i j}^{\prime}=\left\{a_{s} \mid a \in \rho\right.$ and $\left.s=x_{i} \times x_{j}\right\}$ In words, if $\dot{\rho}$ is expressed in column form, $R_{i j}^{\prime}$ is obtained by taking the $i$ th and the $j$-th rows (and merging repeated pairs). Note that $R_{i i}^{\prime} \subseteq I$ and that $R_{i j}^{\prime}=R_{j i}^{T}$, i.e. $R^{\prime}$ is symmetric. 
Some properties of $R^{\prime}$ are proved by the following theorem. Theorem 3.1 The network of relations $R^{\prime}$ defined by (3.2) represents an n-ary relation $\rho^{\prime}$ such that

$$
\rho \subseteq \rho^{\prime}
$$

Furthermore, no network $R^{\prime \prime}$ exists, which represents an n-ary relation $\rho^{\prime \prime}$ such that

$$
\rho \subseteq \rho^{\prime \prime} \subset \rho^{\prime}
$$

Thus, in particular, $\rho^{\prime}=\rho$ if $\rho$ is representable by a network. Broof Formula(3.3) is easy proved, because n-tuples a $\rho$ satisfy network $R^{\prime}$ by construction. We will prove the second part by contradiction. Let a be an n-tuple such that a $\in \rho^{\prime}$ but a $\notin \rho^{\prime \prime}$. Thus some projection b of a does not satisfy a relation of $R^{\prime \prime}$, say $b=a_{S}, S=x_{i} \times x_{j}, b \notin R_{i j}^{\prime \prime}$, while $b \in R_{i j}^{\prime}$ But if the pair $b$ was included in $R_{i j}^{\prime}$ it means that an n-tuple $\bar{a} \in \rho$ exists, such that $\bar{a}_{S}=b$. This is a contradiction, because then $\mathbf{a} \notin \rho$ " while we assumed $\rho \subseteq \rho^{\prime \prime}$.

\section{Q.E.D.}

As an example of the projection procedure let us consider the following relation:

$$
\text { (3.4) } \quad \rho=\left(\begin{array}{lll}
1 & 1 & 2 \\
1 & 2 & 2 \\
1 & 2 & 1
\end{array}\right)
$$

The approximating network is:

$$
\begin{aligned}
& \left.n_{12}^{\prime}=\mid \begin{array}{lll}
1 & 1 & 2 \\
1 & 2 & 2
\end{array}\right)=\left|\begin{array}{ll}
1 & 0 \\
1 & 1
\end{array}\right| ; \quad R_{23}^{\prime}=\left|\begin{array}{lll}
1 & 2 & 2 \\
1 & 2 & 1
\end{array}\right|=\left|\begin{array}{ll}
2 & 0 \\
1 & 1
\end{array}\right| ; \\
& \left.R_{13}^{\prime}=\left(\begin{array}{lll}
1 & 1 & 2 \\
1 & 2 & 1
\end{array}\right)=\mid \begin{array}{ll}
0 & 1 \\
1 & 1
\end{array}\right) ; \quad R_{11}^{\prime}=R_{22}^{\prime}=R_{33}^{\prime}=I
\end{aligned}
$$

The induced $n$-ary relation is :

$$
\text { (3.5) } \quad P^{\prime}=\left(\begin{array}{llll}
1 & 1 & 2 & 1 \\
1 & 2 & 2 & 2 \\
1 & 2 & 1 & 1
\end{array}\right)
$$

A partial ordering among networks of constraints having the same number $n$ of vertices can be introduced in a natural way. The ordering relation is defined 


$$
=u_{4}=
$$

as follows:

$$
\text { (3.6) } R^{\prime} \subseteq R^{\prime \prime} \quad \text { iff } \quad R_{i j}^{\prime} \subseteq \mathbb{R}_{i j}^{\prime \prime} \quad(1, j=1, \ldots, n)
$$

The reflexive, weakly antisymetric and transitive properties for network inclusion descend from the same properties for set inclusion. It is also clear that the set of all networks with $n$ vertices is a lattice under $\subseteq$ because a least $\left(R_{i j}=\emptyset_{i j}\right)$ and greatest $\left(R_{i j}=U_{i j}\right.$ if $\left.i \neq j ; R_{i i}=I\right)$ networks exist. Thus union and intersection between two networks are defined. It is also irmediate to see that

(3.7) $R^{\prime} \subseteq R^{\prime \prime} \quad$ implies $\rho^{\prime} \subseteq \rho^{\prime \prime}$

where $R^{\prime}$ and $R^{\prime \prime}$ represent $P^{\prime}$ and $P^{\prime \prime}$.

We have seen that not all the n-ary relations are representable by a network of constraints. It can also happen that an $n$-ary relation $\rho$ is representable by many distinct networks. For instance, relation (3.1) cen be represented by the networks in both Fig.la and Fig. Ib. Two networks $R^{\prime}$ and $R^{\prime \prime}$ which represent the same n-ary relation $\rho$ are called equivslent.

The next theorem proves the existence of a minimal network $M$ representing $p$. Theorem 3.2 Let $S_{p}$ be the equivalence class of all networks representing the same relation $p$. If

$$
R^{\prime} \in s_{p} \text { and } R^{\prime \prime} \in s_{p}
$$

also

$$
\mathrm{R}=\mathrm{R}^{\prime} \cap \mathrm{R}^{\prime \prime} \in \mathrm{S}_{\mathrm{p}}
$$

(See for instance Fig $l a, b, c$ ). As a consequence, a minimal (with respect to $\subseteq$ ) network $M$ representing $\rho$ exists, and can be obtained from $\rho$ by the projection formula $(3.2)$.

Proof To prove this theorem, we first notice that $R \subseteq R^{\prime}$ and $R \subseteq R^{\prime \prime}$, and 
thus an m-tuple a satisfying $R$ satisfies also $R^{\prime}$ and $R^{\prime \prime}$ for (3.7). Conversely, if a satisfies $R^{\prime}$ and $R^{\prime \prime}$ it satisfies also $R$. In fact, for each subspace $S=X_{i} \times X_{j}$, if $a_{S} \in R_{i j}^{\prime}$ and $a_{S} \in R_{i j}^{\prime \prime}$ then $a_{S} \in R^{\prime} \cap R^{\prime \prime}=R$ by the definition of intersection. Finally, the network obtained by formula (3.2) must be minimal: if any pair $b$ is erased by any relation $R_{i j}$, the represented relation $\rho$ is changed.

$$
\text { Q.E.D. }
$$

Given an n-ary relation $\rho$ representable with a (minimal) network of constraints $M$, and a subspace $S=X_{I} \times \ldots \times X_{m}$ of $X$, one could ask if the projection $\rho_{S}$ of $p$ : (3.9) $\rho_{S}=\left\{a_{S} \mid a \in \rho\right\}$

is representable with a network of m nodes. Interestingly enough, in the general case the answer is no, and a counterexample is given in Fig. 2 . There, relation $\rho$ is

$$
\begin{aligned}
P= & \left(x_{1,1}, x_{2,1}, x_{3,1}, x_{4,1}\right),\left(x_{1,1}, x_{2,2}, x_{3,2}, x_{4,2}\right), \\
& \left.\left(x_{1,2}, x_{2,1}, x_{3,1}, x_{4,3}\right)\right\}
\end{aligned}
$$

If $S=X_{1} \times X_{2} \times X_{3}, P_{S}$ is given by (3.4). But, as we saw, (3.4) is not representable with a three-vertex network. If $\rho_{S}$ is representable for all $s$, then $\rho$ and all networks representing $\rho$ are called decomposable. If not, the next theorem gives the best approximating network for $\mathrm{P}_{\mathrm{S}}$.

Theorem 3.3 The best, minimal approximating network of $\rho_{S}$ is the complete subnetwork $M^{S}$ of $M$ corresponding to the set of vertices $\bar{S}=\left\{X_{1}, \ldots, X_{m}\right\}$. Proof This property descends immediately from theorem (3.1) and from the fact that if $T=X_{j} \times X_{j}$ is any bidimensional subspace of $S$, we have:

$$
\left(\rho_{S}\right)_{T}=\rho_{T}
$$


In the remainder of the paper we will be often concerned in proving that a network $R$ is minimal: $M=R$. The next theorem gives a characteristic condition for $R$ to be minimal.

Theorem 3ele A necessary and sufficient condition for a network $R$ to be minimal, is that if a pair $b$ satisfies the generic reletion $R_{i j}$, an $n$-tuple a satisfying $R$ exists, such that $a_{s}=b, s=x_{i} \times x_{j}$.

Proof Necessity. If $R$ is minimal and $b \in R_{i j}$, an $n$-tuple $a \in P$ must exist such that $a_{S}=b$, because otherwise $R^{\prime}$ with $R_{i j}^{\prime}=R_{i j}-\{b\}$ would be equivalent to $R$ and smaller.

Sufficiency Under our assumption, no pair can be erased from any $R_{i f}$ still obtaining an equivalent network. Thus $R$ is minimal.

Q.E.D.

The last theorem can be modified as follows.

Corollary Given any relation $R_{i j}$ of $R$, if whenever a pair $b$ belongs to $R_{i j}$, an $n$-tuple a satisfying $R$ exists such that as $=b, S=X_{i} \times X_{j}$, then $R_{i j}=M_{i j}$.

Proof This statement follows from the previous theorem and from the equivalence of $R$ and $M$.

\section{Q.E.D.}

The above theorem shows that a minimal network of constraints is perfectly explicit: as far as the pair of variables $x_{i}$ and $x_{j}$ is concerned, the rest of the network does not add any further constraint to the direct constraint $M_{i j}$ • Minimal networks are likely to represent an $n$-ary relation in a redundant way. In our application, for instance, we expect to define constraints almost only between geometrically adjacent elements. As a result, the density d of connections (defined as the average number of arcs per vertex) should be bounded,like 
that of a planar graph, $\mathrm{d}<3$ * or at most should grow logarithmically with the number of vertices, but not linearly like in a complete graph.

From the above reasoning, should be clear that, in our application, networks of constraints will never be given or stored as minimal networks. Furthermore, the trivial way of getting the minimal network, i.e., generating the n-ary relation $\rho$ from the given $R$, and then $M_{1}$ from $\rho$ with (3.2) will be impossible in continuous cases and always practically infeasible. Therefore the problem of computing $M$ from $R$ in an economic way is nontrivial. On the other hand we can show that most of the practical problems arising from the use of networks of constraints can be naturally reduced to the central problem of deriving $M$. In fact, in the first scenario described in the introduction, if $V_{O k}(k=1, \ldots, m)$ are the fundamental vectors corresponding to the already determined values of the first $m$ variables, the intersection of images

$$
R_{O p}=\sum_{k=0}^{m} V_{O k} M_{k p}
$$

clearly represents the set of allowed values for the p-th variable. In scenario b), if we want to eliminate a variable (related to $\mathrm{m}$ others variables) using a sequential optimization method, we must optimize the objective function separately for all the feasible m-tuples of related variables. The set of those $m$-tuples is $p_{S}$, if $S$ is the subspace of the related variables. By theorem (3.3) $\mathrm{M}^{\mathrm{S}}$ is the best approximating network of $\rho_{\mathrm{S}}$. Finally, in scenario (c) the minimal subnetwork $\mathrm{M}^{\bar{S}}$ corresponding to the parts which are not missing constitutes the best reduced model.

* In a planar graph, the number $n$ of vertices is related to the number $a$ of arcs by the relation

$$
a \leq 3 n-6
$$

Equalj.ty is achieved if all the faces are triangular. 


\section{Approximate solution of the central problem}

In this section we consider the problem of computing the minimal network equivalent to a given network. No exact general algorithm, besides complete enumeration, was found. However, an approximate solution is given, which generates an equivalent "closed" network.

In a generic network of constraints, a certain pair $\left(x_{i, r}, x_{j, s}\right)$ can be allowed by the direct relation $R_{i j}$ (or also by $R_{j 1}, R_{1 i}$ and $R_{j j}$ ) but can be actually forbidden because it is not possible to give to all the other variables any set of values allowed by all the constraints. To recognize such pairs and erase them, namely to make explicit the global constraint, is the essence of the central problem. The central problem, in its generality, is very difficult. Graph coloring problems, for instance, are very neatly represented by networks of constraints: relations are all of the type U - I, i.e., all pairs allowed except those of the saine colour. The number of allowed colours (i.e. the cardinality of sets $x_{i}$ ) and the topology of the graph characterize the particular problem. For instance, Fig. 3 shows the network of constraints representing the problem of coloring a tetrahedron with three colours: an impossible task. However, it is difficult to recognize it with a sequence of local examinations of the network, and without "higher order" reasonings. Needless to say, no hope exists to extend such tricks to the general case. Therefore we look for an approximation of the minimal network $M$, i.e., a network $Y$ which is as explicit as possible and still computable with local operations.

Let us consider an ordered pair of values

$b=\left(x_{i, r}, x_{j, s}\right)$ 
and a path *

$$
P=\left(v_{i}=v_{i}, \ldots, v_{i p}, \ldots, v_{i_{m}}=v_{f}\right) \quad m>1
$$

in the complete network $R$ from vertex $V_{i}$ to vertex $V_{j}$. The pair $b$ is allowed by the path $p$ if the variables

$$
x_{i}=x_{i_{0}}, \ldots, x_{i_{p}}, \ldots, x_{i_{m}}=x_{j}
$$

can be given suitable values

$$
x_{i, r}=x_{i_{0}, r_{0}}, \ldots, x_{i_{p}, r_{p}}, \ldots, x_{i_{m}, r_{m}}=x_{j, s}
$$

which satisfy the relations

$$
R_{i_{0} i_{1}}, \ldots, R_{i_{p-1} i_{p}}, \ldots, R_{i_{m-1} i_{m}}
$$

along the spath $P$. Note that the same vertex $V_{k}$ can occur in a path any number of times, and different values can be given to its variable $x_{k}$ for each occurrence. A pair $b$ is called legal if it is allowed by all the paths $P$ from $\mathrm{V}_{i}$ to $\mathrm{V}_{j}$. We will see that the property of being legal is decidable in a finite number of steps. Finally, a network is called closed if any pair $b$ which is not legal is also not allowed by the direct relation $R_{i j}$.

It is clear from the definition that minimal networks are closed. The converse is, in general, not true. For instance, the network in Fig. 3 (representing the uncolorable tetrahedron) is closed but not minimal. This also means that many closed networks equivalent to a given network may exist. Given a network $R$, its closure $Y$ is defined as the largest closed network not larger then $R$ but equivalent to $R$. The next theorem proves the uniqueness of the closure. Theorem 1.21 The set of closed networks not larger than $R$ but equivalent to $R$ (which is ordered under $\subseteq$ ) has a largest element $Y$. Therefore $Y$ is the only closure of R.

\footnotetext{
* A path in $R$ is any sequence of vertices. A vertex can occur more then once in a path, even in consecutive positions.
} 
Proof We must prove that the union of two closed networks $Y^{\prime}$ and $Y^{\prime \prime}$ both not larger than $R$ but equivalent to $R$ is a closed network $Y$ not larger than $R$ but equivalent to $R$. In fact from $R \geq Y^{\prime}$ and $R \geq Y^{\prime \prime}$ we have $R \geq Y^{\prime} U Y^{\prime \prime}=Y$. From $R \geq Y \geq Y^{\prime}, R$ equivalent to $Y^{\prime}$ and (3.7) twice, we have $Y$ equivalent to R. Let $Y_{i, j, r s}=1$. Then for $Y=Y^{\prime} \cup Y^{\prime \prime}$ either $Y_{i j, r s}^{\prime}=1$ or $Y_{i j, r s}^{\prime \prime}=1$ or both, say $Y_{i j, r s}^{\prime}=1$. Then the pair $b=\left(x_{1}, r, x_{j, s}\right)$ is allowed by $P$ in $Y^{\prime}$ for closure. Thus $b$ is allowed by $P$ also in $Y$, because the same set of path values satisfying $Y^{\prime}$ satisfies also $Y$, for $Y^{\prime} \subseteq Y$. Q.E.D.

The closure $Y$ of a network $R$ can be characterized as being the solution of the following system of equations. (4.I) $Y_{i j}=\sum_{k=1}^{n} R_{i k} Y_{k j}+d_{i j}$ where

$$
d_{i j}=I_{i j} \quad \text { if } i=j \quad ; \quad d_{i j}:=U_{i j} \text { otherwise. }
$$

A network of relations $Y$ is called a solution of system (4.1) iff:

i) The relations $Y_{i j}$ satisfy equations (4.I).

ii) No other network $Y^{\prime}$ exists, such that $Y^{\prime}$ satisfies equations (4.I) and $Y^{\prime} \supseteq Y$.

Note that condition (ii) is necessary for ruling out solutions which are not equivalent to $R$ (like the trivial case $Y_{i j}=\phi_{i j}$ ) and that it does not imply uniqueness of the solution a priori. We can prove the following theorem. Theorem 4.2 Any network $Y$ which satisfies system (4.1) is:
a) Not larger than $R$.
b) Closed. 
If furthermore $Y$ is a solution of system (4.1), then $Y$ is:

c) Equivalent to $R$

d) The closure of $R$

Therefore only one solution exists.

Proof a) From (4.1) we have

$$
Y_{j j} \subseteq I_{j j}
$$

Thus, by the monotonicity property of composition:

$$
R_{i j} Y_{j j} \subseteq R_{i j}
$$

And finally, from $(4.1)$

$$
Y_{i j} \subseteq R_{i j} Y_{j j} \subseteq R_{i j}
$$

b) Given any path $P$ we will prove that if $Y_{i j, r s}=I$ and $Y$ satisfies equations (4.1) then the pair $b=\left(x_{1, r}, x_{j, s}\right)$ is allowed by $P$ in $R$, i.e. the relations of $R$ along $P$ can be satisfied. We will prove this result by induction on the length $m$ of the path $P$. If $m=1$ the proof is trivial, because $Y_{i j}$ is the only relation which must be satisfied. If the result is true for every path of length $(\mathrm{m}-1)$ it is true also for paths of length $\mathrm{m}$. In fact, from (4.1) we have:

$$
Y_{i j}=Y_{i_{0} i_{m}} \subseteq R_{i_{0} i_{1}} Y_{i_{1} i_{m}}
$$

Therefore at least one value $x_{i_{1}, r_{1}}$ must exist, such that $R_{i_{0} i_{1}, r_{0} r_{1}}=1$ and $Y_{i_{I} i_{m}, r_{I} r_{m}}=1$. Thus relation $R_{I_{0} i_{I}}$ is satisfied by $\left(x_{i_{0}}, r_{0}, x_{i_{I}, r_{I}}\right)$, while $Y_{i_{1} i_{m}}, r_{I} r_{m}=I$ implies that the $m-1$ relations $R_{i_{1} i_{2}}, \ldots, R_{i_{m-1} i_{m}}$ can be aatisfied according to the induction assumption.

c) If an $n$-tuple satisfies $Y$, it satisfies also $R$, because $Y \subseteq R$ for a). Conversely, let $a=\left(x_{1}, r_{1}, \ldots, x_{n, r_{n}}\right)$ be an $n$-tuple satisfying network $R$, namely $R_{i j, r_{i} r_{j}}=I(i, j=1, \ldots, n)$. We will prove that a satisfies network $Y$ too. In fact, if we assume $Y_{i j, r_{i} r_{j}}=0$ for some $i, j$, it is possible 
to find a larger network $Y^{\prime} Y$ (against ii)) satisfying (4.I) and

such that $Y_{i j, r_{i} r_{j}}=1$. For proving $i t$, let

$$
Y_{i j, r_{i} r_{j}}^{O}=1 ; Y_{i j, r s}^{0}=Y_{i j, r s} \text { if } r \neq r_{i} \text { and } s \neq r_{j}
$$

It is immediate to see that equations

$$
Y_{i j, r_{i} r_{j}}^{0}=\bigwedge_{k=1}^{n} \bigvee_{t=1}^{N_{k}}\left(R_{i k, r_{i} t} \wedge \mathrm{Y}_{k j, t r}^{0}\right) \wedge d_{i j, r_{i} r_{j}}
$$

are satisfied, while from $Y$ satisfying (4.I) we have:

$$
r_{i j, r s}^{0} \subset \bigwedge_{k=1}^{n} \bigvee_{t=1}^{N k}\left(R_{i k, r t} \wedge r_{k j, t s}^{0}\right) \wedge d_{i j, r s} \quad\left(i, j=1, \ldots, n ; j \neq i \neq r_{i}, j \neq r_{j}\right)
$$

$$
Y_{i j}^{0} \subseteq \sum_{k=1}^{n} R_{i k} Y_{k j}^{0}+d_{i j}
$$

Now, if we compute iteratively

we will have

$$
\mathrm{Y}_{i, j}^{p}=\sum_{k=1}^{n} R_{i k} Y_{k j}^{p-1}+\alpha_{i j}
$$

$$
Y^{O} \subseteq Y^{I} \subseteq \cdots \subseteq Y^{p}
$$

from monotonicity of intersection and composition. Thus for some $q$ we will have

$$
\mathrm{Y}^{\mathrm{q}}=\mathrm{Y}^{\mathrm{q}+1}=\mathrm{Y}^{\dot{1}}
$$

satisfying (4.1) and such that $Y^{i} \supseteq Y^{0} \supset Y$.

d) Let $\bar{Y}$ be the closure of $R$. From the definition of closed network, applied to paths of length two, we have:

$$
\bar{Y}_{i j} \subseteq \bar{Y}_{i k} \bar{Y}_{k j}+d_{i j}
$$

Then from $Y \subseteq R$

$$
\bar{y}_{i j} \subseteq R_{i k} \bar{Y}_{k j}+d_{i j}
$$

Summing up, we have::

$$
\bar{Y}_{i j} \subseteq \sum_{k=1}^{n} R_{i k} \bar{Y}_{k j}+d_{i j}
$$

Actually, we must have equality, i.e., (4.1), because otherwise with the iterative method shown in part c) a network $\bar{Y}^{\prime}$ can be found which satisfies (4.1). Then 
the proof of part $c$ ) shows that a network $\vec{Y}^{\prime} \leq \vec{Y}^{\prime} \subseteq \bar{Y}$ can be found which satisfies ( 4.1 ) (and thus is closed and not larger than $R$ ) and is equivalent to $R$. The existence of $\vec{Y}^{\prime \prime}$ would contradict the maximality of $\bar{Y}$ proved in theorem(4.1). Thus $\bar{Y}$ satisfies $i)$. On the other hand, if $Y$ is any solution, we must have $\bar{Y} \subseteq Y$ for the same reason. Thus $\bar{Y}$ satisfies $i i)$ and is the only solution.

\section{Q.E.D.}

The next corollary gives a simple way for characterizing a closed network. Corollary A necessary and sufficient condition for a network $Y$ to be closed is to satisfy the following system of equations:

(4.2) $Y_{i j}=\sum_{k=1}^{n} Y_{i k} Y_{k j}+d_{i j} \quad(i, j=1, \ldots, n)$

Proof- According to theorem (4.2b), if $Y$ satisfies system (4.2) Y is closed. Conversely, if $Y$ is closed, it must be the closure of itself and thus by theorem (4.2d) must satisfy equations (4.2).

$$
\text { Q.E.D. }
$$

The next theorem proves a useful property of closed networks.

Theorem 4.3 In a closed network $Y$, the loop relations and the relations among different yertices satisfy the following equation:

$$
\text { (4.3) } Y_{i i}=Y_{i j} U_{j i}+I_{i i} \quad(i, j=1, \ldots, n)
$$

Proef From $(4.2)$ we have

$$
Y_{i i} \subseteq Y_{i j} Y_{j i}+I_{i i}
$$

and from monotonicity

$$
Y_{i i} \subseteq Y_{i j} U_{j i}+I_{i i}
$$

Furthermore, from (4.2) we have

$$
Y_{i j} \subseteq Y_{i i} Y_{i j}
$$

In binary form we have:

$$
Y_{i j, r s} \subset Y_{i i, r r} \wedge Y_{i j, r s} \quad\left(r=1, \ldots, N_{i} ; s=1, \ldots, N_{j}\right)
$$


or, from a truth table

$$
Y_{i j, r s} \subset Y_{i i, r r}
$$

Making the union with respect to $\mathbf{s}$ :

$$
\bigvee_{s=1}^{N} Y_{i j, r s} \subseteq Y_{i i, r r} \quad\left(r=1, \ldots, N_{i}\right)
$$

Equivalently we can write

$$
Y_{i j} U_{i i}+I_{i i} \subseteq Y_{i i}
$$

Therefore we have

$$
Y_{i i}=Y_{i j} U_{j i}+I_{i i}
$$

Q.E.D.

Given a network $\mathrm{R}$ with $\mathrm{n}$ vertices we can give an algorithm for computing its closure $\mathrm{Y}$.

\section{Algorithin $C$}

Step 1 $\quad Y^{O}=R$

Step2 Execute next step for $k=1, \ldots, n$

Step_3 (4.4) $y_{i j}^{k}=Y_{i j}^{k-1}+y_{i k}^{k-1} Y_{k k}^{k-1} Y_{k j}^{k-1} \quad(i, j=1, \ldots, n)$

Step 4 If $Y^{n} \neq Y^{0}$ then let $Y^{O}=Y^{n}$ and go to step 2 ; else let $Y=Y^{n}$ and stop.

Theorem 4.4 Algorithm $C$ computes the closure $Y$ of $R$. In particular, if $r_{i j, r s}^{n}=I$ in the network $Y^{n}$ obtained at the end of the first iteration, then pair $\left(x_{i, r}, x_{j, s}\right)$ is allowed by all the paths from $V_{i}$ to $V_{j}$ in $R$.

Proof We will prove that i) $Y$ is not larger than and equivalent to $R$; ii) $Y$ is closed ; $i i i)$ for every closed network $Y^{\prime}$ equivalent to $R$ and not larger than $R$ we have $Y^{\prime} \subseteq Y \subseteq R$.

i) Each application of step 3 produces a network $\mathrm{Y}^{\mathrm{k}}$ equivalent to the precedent $Y^{k-1}$. In fact, clearly $Y^{k} \subseteq y^{k-1}$. On the other hand, if the second term in the right member of $(4.4)$ :

$$
\bigvee_{t=1}^{N} y_{i k, r t}^{k-1} \wedge y_{k k, t t}^{k-1} \wedge \mathrm{x}_{k j, t s}^{k-1}=0
$$

is zero for some $r, s$ it means that no value for $x_{k}$ can be found which 
satisfies relations $\mathrm{Y}_{i k}^{k-1}, \frac{1}{k k}_{k-1}^{k}$ and $Y_{k j}^{k-1}$ for $x_{i}=x_{i, r}$ and $x_{j}=x_{j, s}$. Thus no $n$-tuple is excluded by $Y^{k}$ which is not excluded by $Y^{k-1}$. Therefore, from transitivity of equivalence and inclusion, $Y$ is equivalent to $\mathrm{H}$ and $\mathrm{Y} \subseteq R$. ii) We will prove that if a pair is allowed by $Y^{\mathbf{n}}$ then is allowed by all paths in $y^{0}$. We assume that when step 3 was executed $(k-1)$ times, if a pair $\left(x_{j, r}, x_{j, s}\right)$ is allowed by the relation $Y_{i j}^{k-1}$, then it is allowed also by all the paths in $\mathrm{Y}^{0}$ with extrema in $\mathrm{V}_{i}$ and $\mathrm{V}_{j}$ and having all the intermediate vertices with indexes $\leq k-1$. If $k=1$ the assumption is trivially true. We will prove the same property for $k$ after the $k$ th execution. Let us consider ans path $\mathrm{P}$ from $\mathrm{V}_{i}$ to $\mathrm{V}_{j}$ having intermediate vertices with indexes $\leq \mathrm{k}$. If vertex $\mathrm{v}_{\mathrm{k}}$ does not belong to this path, the induction step is proved. If it does, path $P$ can be decomposed in three paths:

a) a path from $V_{i}$ to $V_{k}$

b) a finite (possibly zero) number of circuits from $V_{k}$ to $V_{k}$

c) a path from $v_{k}$ to $v_{j}$

All those paths have intermediate vertices with indexes $\leq k-I$. According to the $\operatorname{step}\left(4 \cdot 4_{4}\right)$, if $y_{i j, r s}^{k}=1$, then a value $x_{k, t}$ can be found such that $Y_{i k, r t}^{k-1}=1, Y_{k k, t t}^{k-1}=1$ and $Y_{k j, t s}^{k-1}=1$. Thus by the jnduction assumption we can give to all the intermediate variables of paths a),b) and c) suitable values which satisfy the corresponding constraints in $Y^{0}$. When the algorithm stops, we have $Y^{n}=Y^{0}=Y$ and thus $Y$ is closed. iii) Let $Y^{\prime} \subseteq R$ be an: closed network equivalent to $R$. We will have $Y^{\prime} \subseteq Y^{k}$ for all $k$ and for all iterations of algorithm $C$. Thus also $Y^{\prime} \subseteq Y$. Inductively, let us assume that $Y^{\prime} \subseteq \mathrm{Y}^{\mathrm{k}-1}$ before the execution of step 3: This is certainly true for the first execution of step 3 in the first iteration: $Y^{0}=R$. Then $Y^{*} \subseteq Y^{k}$. In fact, if network $Y^{\prime}$ is closed, it satisfies 
equations (4.2) for the corollary to theorem (4.2). In particular, we have:

$$
\mathrm{Y}_{i j}^{\prime} \subseteq \mathrm{Y}_{i k}^{\prime} \mathrm{Y}_{k j}^{\prime}+\mathrm{d}_{i j} \quad ; \quad \mathrm{Y}_{\mathrm{kj}}^{\prime} \subseteq \mathrm{y}_{\mathrm{kk}}^{\prime} \mathrm{Y}_{\mathrm{kj}}^{\prime}
$$

and therefore for the monotonicity of composition and intersection:

$$
\mathrm{Y}_{i j}^{\prime} \leq \mathrm{y}_{i k}^{\prime} \mathrm{y}_{k \mathrm{k}}^{\prime} \mathrm{Y}_{k j}^{\prime}+d_{i j}
$$

and thus

$$
\text { (4.4) } \quad \dot{Y}_{i j}^{\prime}=Y_{i j}^{\prime}+Y_{i k}^{\prime} Y_{k k}^{\prime} Y_{k j}^{\prime}+d_{i j}
$$

But from the induction assumption and monotonicity we have:

$$
\text { (4.5) } \mathrm{Y}_{i j}^{\prime}+\mathrm{y}_{i k}^{\prime} \mathrm{Y}_{\mathrm{kk}}^{\prime} \mathrm{y}_{k j}^{\prime}+\mathrm{d}_{i j} \subseteq \mathrm{y}_{i j}^{\mathrm{k}-1}+\mathrm{Y}_{i k}^{\mathrm{k}-1} \mathrm{Y}_{\mathrm{kk}}^{\mathrm{k}-1} \mathrm{Y}_{\mathrm{kj}}^{\mathrm{k}-1}+\mathrm{d}_{i j}
$$

Now note that the $d_{i, j}$ term in the right member is redundant because if $i \neq j$

then $d_{i j}=U_{i j}$ and if $i=j$ then $d_{i j}=I_{i i}$ and $Y_{i j}^{k-1} \subseteq R_{i i} \subseteq I_{i i}$.

Thus from $(4.3)(4.4)$ and $(4.5)$ we have

$$
y_{i j}^{\prime} \subseteq y_{i j}^{k}
$$

\section{Q.E.D.}

It is interesting to see how the number of iterations required by algorithm 6 is dependent on the order of vertices in step 2. For instance, in network $R$ in $\mathrm{Fig} \cdot(4 \mathrm{a})$, if $\mathrm{k}=1,2,3,4$ then only one iteration is necessary for finding the closure $Y\left(Y_{i j}=\phi_{i j}\right)$. If $k=4,1,2,3$ then two iterations are necessary. Fig. (4b) shows the network $Y^{\dagger}$ obtained at the end of the first iteration. According to the above theorem, if $Y_{i j, r s}^{n}$ in the network $Y$ obtained at the end of first iteration, then the pair $\left(x_{i, r}, x_{j, s}\right)$ is allowed by all paths in $R$. For instance in the above example for $i=1, j=2$ and $r=s=1$ and for path $P=\left(V_{1}, V_{3}, V_{4}, V_{2}\right)$, values $x_{1,1}, x_{3,1}, x_{4,1}$ and $x_{2,1}$ satisiy the three relations $R_{13}, R_{34}, R_{42}$ along $P$. Note how this condition is pot sufficient for $Y^{\prime}$ being closed. Thus in general one iteration of algorithm $C$ is not sufficient. On the other hand, each iteration of algorithm $C$ produces an equivalent, strictly smaller network, and thus convergence is assured. 


\section{Exact solution of the central problem for particular classes of networks}

In the last section we have been able to give only an approxinate solution to the central problem in the general case. A closed network instead of the minimal network was obtained. We can now ask if there are particular cases in which a closed network is always minimal.

In general, given a network $R$ and a pair of vertices $V_{i}, V_{j}$ we are interested in knowing if the relation $Y_{i j}$ of the closure $Y$ of $R$ coincides with the relation $M_{i j}$ of the minimal network equivalent to $R$. In this case, network $R$ will be called reqular with respect to pair $V_{1} V_{j}$. If $R$ is regular with respect to all pairs of vertices, it will be called regular. Thus for a regular network $R$ we have $Y=M$. In this section we will see that interesting classes of networks are regular.

We can determine regular classes of networks in essentially two ways: either constraining the topology of the network or restricting the type of allowed relations. We will consider the former case first.

A symmetric * series-parallel network (spn) with respect to a pair of vertices $V_{i} V_{j}$ is usually defined recursively as follows:

a) A complete symmetric network with two vertices $V_{i}$ and $V_{j}$ is a spn. b) Given two spn's with respect to $v_{j}^{\prime} v_{j}^{r}$ and $v_{i}^{\prime \prime} v_{j}^{\prime \prime}$, the network obtained letting $v_{j}^{p}$ and $v_{i}^{\prime \prime}$ coalesce is a spn with respect to $v_{i}^{\prime} v_{j}^{\prime \prime}$.

* In what follows, symmetry will be almost always required, since a minimal network is obviously symmetric, while symmetry is not assured for a closed network. On the other hand, an equivalent, symmetric and not larger network $R^{\prime}$ can be immediately computed from any $R$ with the formula: $R_{i j}^{\prime}=R_{i j}+R_{j i}^{T}$. Its closure $Y$ is then symmetric, as is obvious from algorithm $C$. 
c) Given two spn's with respect to $V_{i}^{\prime} V_{j}^{\prime}$ andV $v_{i}^{\prime \prime} V_{j}^{\prime \prime}$, the network obtained letting $v_{i}=v_{i}^{\prime}=v_{i}^{\prime \prime}$ and $v_{j}=v_{j}^{\prime}=v_{j}^{\prime \prime}$ is a spn with respect to $v_{i} v_{j}$. As usual, all missing arcs $V_{i} V_{j}$ are assumed to correspond to the universal relations $U_{i j}$.

In the last, section we saw that in a closed network $Y$ a relation $Y_{i j}$ makes explicit the constraints given by all the paths from $V_{i}$ to $V_{j}$. The next lemma proves that a closed network has the same property for all the subnetworks which are series parallel with respect to $V_{i} V_{j}$.

Iemma 5.1 Let $Y$ be a symmetric closed network, and let $\mathrm{R}$ be any subnetwork of $Y$ which is a spn with respect to $V_{i} V_{j}$. Let $M$ be the minimal network equivalent to $R$. We have:

(5.1) $Y_{i j} \subseteq M_{i j}$

Proof According to the corollary to theorem (3.4) we must prove that if $Y_{i j, r s}=I$ then an $m$-tuple satisfying $R$ ( $R$ has $m$ vertices) such that $x_{i}=x_{i, r}$ and $x_{j}=x_{j, s}$ can be found. We will use induction applied to each step of the recursive definition of a spn: we assume that the property is true for the component networks and we prove it for the resulting network. For steps of type a), if $Y_{i j, r s}=I$ then $Y_{j i, s r}=1$ for symmetry, while $Y_{i i, r r}=I$ and $Y_{j j, s s}=1$ for $(4.3)$. For steps of type $\left.b\right)$, let $V_{k}$ be the vertex in the middle of the series. If $Y_{i, j, r s}=I$ and $Y$ is closed, then a value $x_{k}=x_{k, t}$ exists such that $Y_{i k, r t}=I$ and $Y_{k j, t s}=I$ and therefore the recursive assumption can be applied because no constraint exists in $R$ between two vertices in different components. In definition c), the recursive assumption can be directly applied to both components.

$$
\text { Q.E.D. }
$$

As an example of application of this theorem, let us consider again the 
coloring problem represented by the closed but not minimal network $Y$ in Fig. 3 . In Fig. 5 a we have a subnetwork $R$ which is a spn with respect to all pairs of vertices except $\mathrm{V}_{2} \mathrm{~V}_{3}$. In Fig. $4 \mathrm{~b}$ we have the minimal network $M$ equivalent to R. Note that $Y_{i j} \subseteq M_{i j}$ (in fact, $Y_{1 j}=M_{i j}$ ) for all pairs except $V_{2} V_{3}$. The next theorem proves the regularity of some classes of networks. Theorem 5.1 a) Tree networks $\because$ are regular. b) Symmetrical series parallel networks with respect to a pair $V_{i} V_{j}$, possibly with trees rooted at any vertex, are regular with respect to $v_{i} V_{j}$. Proof a) Let $R$ be a tree network, and let $Y$ be its closure. Given a pair $V_{i} V_{j}$, let $P$ be the only connecting path in $R_{\text {. If }} Y_{i j, r s}=1$, suitable values can be given to the vertices of $P$ which satisfy the relations of $Y$ along $P$, since $Y$ is closed. It is now easy to see that suitable values can also be given to the other variables. It is sufficient to evaluate them following the tree structure of $R$, i.e., in such a way that each new vertex $V_{k}$ to be evaluated is adjacent in $R$ to one already evaluated vertex $V_{h}$ (more than one vertex is not possible: a circuit would be present). Inductively, let $x_{h, t}$ be the value already assigned to $x_{h}$. To $x_{k}$ we can assigne any value $x_{k, s}$ such that $Y_{h k, t s}=1$. Such a value must exist, because otherwise $Y_{h h, t, t}=0$ for theorem (4.3) and thus $Y_{p h, t t}=0$ for all $p$ (again for the same theorem) so that the value $x_{h}, t$ could not have been given previousiy to $x_{h}$ according to this procedure. At the end, all the variables have been evaluated and satisfy the $Y$ constraints along the tree, and thus also all the $R$ constraints, because $Y \subseteq R$ for theorem (4.la), and $R$ has constraints only along the tree. Therefore also $Y$ is satisfied, because $Y$ and $R$ are

* As with spn, the branches of the tree correspond to symmetric, 2-vertex networks. 
equivalent. Thus $Y$ is mininal and $R$ is regular.

b) Let $R$ be the spn and let $Y$ be its closure. Let $Y^{\prime}$ be the subnetwork of $Y$ topologically equivalent to $R$. We have $Y \subseteq Y^{\prime} \subseteq R$. But $Y$ is equivalent to $R$ and thus also $Y^{\prime}$ is equivalent to both $Y$ and $R$ from (3.7) twice. Now let $M$ be the minimal network equivalent to $Y^{\prime}$ and thus to $Y$ and $R$. For Lemm (5.I) we have $Y_{i j} \subseteq K_{i j}$. But $H$ and $Y$ are equivalent and $M$ is minimal. Thus $V_{i j}=Y_{i j}$ and $R$ is regular with respect to $V_{i} V_{j}$. If trees are present at, some vetices of the spn, we can find a feasible n-tuple evaluating the vertices of the spn first, and then evaluating the vertices of each tree as in in part a) of this theorem

$$
\text { Q.F.D. }
$$

In the remainder of this section, we want to determine a class of regular networks by restricting the type of allowed relations. We need the following definition. A network $R$ such that relations $R_{i k}$ and $R_{k i}(i=1, \ldots, n ; i \neq k$ ) fxm a distributive set of relations (see condition (2.1)) for all $k$, is called a distributive network. We can now prove the following theorem. Theorem 5.2 A closed, distrioutive network $Y$ is decomposable. Furthermore, its symetrization

$$
Y_{i j}^{i}=Y_{i j}+Y_{j i}^{T} \quad(i, j=1, \ldots, n)
$$

is minimal. Thus in particular if $Y$ is symetric, it is minimal. Proof We will prove first that if variables $x_{1}, \ldots, x_{k-1}$ can be given values $x_{1, r_{1}}, \ldots, x_{k-1, r_{k-1}}$ which satisfy relations $Y_{i j}(i, j=1, \ldots, k-1)$, a value $x_{k, r_{k}}$ can be given to variable $x_{k}$ which, together with the previous values, satisfy relations $Y_{i j}(i, j=1, \ldots, k)$. From the assumption we have $Y_{i j, r_{i} r_{j}}=1$ or equivalently
$\quad V_{O i} Y_{i j} V_{j 0}=U_{0 O} \quad i, j=I, \ldots, k-1$

where the fundamental vectors $\mathrm{V}_{\mathrm{Oi}}$ and $\mathrm{V}_{30}$ are defined as 


$$
v_{0 i, r}=1 \text { and } v_{10, r}=1 \text { iff } r=r_{1}
$$

From the corollary to theorem (4.2) we have

$$
Y_{i j} \subseteq Y_{i k} Y_{k j} \quad i, j=1, \ldots, k-1
$$

From monotonicity, we obtain:

$$
V_{0 i} Y_{i k} Y_{k j} V_{j 0}=U_{00}
$$

Summing with respect to $i$ and $j$ :

$$
\sum_{i=1}^{k-1} \sum_{j=1}^{k-1} v_{0 i} Y_{i k} Y_{k j} v_{j 0}=U_{00}
$$

Applying distributivity (2.1) we have

$$
\left(\sum_{i=1}^{k-1} v_{0 i} Y_{i k}\right)\left(\sum_{j=1}^{k-1} Y_{k j} v_{j 0}\right)=U_{00}
$$

Then a value $x_{k, r}$ can be found, such that

$\left(\sum_{i=1}^{k-1} V_{0 i} Y_{i k}^{k}\right), r_{k}=1$ and $\left(\sum_{j=1}^{k-1} Y_{k j} V_{j O}\right), r_{k}=1$

or, completely in binary form,

$$
Y_{i k, r_{i} r_{k}}=1 \quad \text { and } \quad Y_{k j, r_{k} r_{j}}=1 \quad i, j=1, \ldots, k-1
$$

Therefore, from theorem (4.3) we have

$$
\mathrm{Y}_{\mathrm{kk}, \mathrm{r}_{\mathrm{k}} \mathrm{r}_{\mathrm{k}}=1}=1
$$

Finally, adding the inductive assumption, we have

$$
Y_{i j, r_{i} r_{j}}=1 \quad 1, j=1, \ldots, k
$$

Observing that the ordering of variables is imaterial and using the above proof as induction step, we have show that if a $\mathrm{k}$-tuple b satisfies any complete subnetwork $Y^{5}$ of $Y, \bar{S}=\left\{v_{i_{1}}, \ldots, v_{i_{k}}\right\}$, at least one n-tuple a exists, whose projection on $S$ is $b$, which satisfies $Y$. On the other hand, if $\mathrm{b}$ does not satisfy $\mathrm{Y}^{5}$, a does not satisfy $\mathrm{Y}$ by definition. Thus the projection $P^{S}$ of the n-ary relation $P$ represented by $Y$ is representable by $Y^{5}$ and therefore $Y$ is decomposable. Furthermore, $Y^{\prime}$ is minimal. In if $Y_{i j, r s}^{p}=1$ the unordered pair $b=\left(x_{i, r}, x_{j, s}\right)$ satisfies the two-vertex 
subnetwork $Y^{\bar{S}}, \quad \bar{s}=\left\{X_{i}, X_{j}\right\}$. Then by the first part of this theorem an n-tuple a exists, such that a satisfies $Y$ and $a_{S}=b$. But $Y$ is equivalent to $Y^{\prime}$ and thus a satisfies $Y^{\prime}$ as well. Therefore $Y^{\prime}$ is minimal for theorem (3.4).

\section{Q.E.D.}

In what follows, it is convenient to consider a particular case in which a slightly different distributive property holds. Given a network $R$, let us consider the set $D$ of all the relations equal to all the possable expressions obtiined by combining relation $\mathrm{R}_{i j}$ with the operations of intersection and composition. If in $\mathrm{D}$ right and left distributivity of composition over intersection always holds, $R$ is called star-distributive. In this case, given any expression, it can always be reduced to a sum of products using distributivity. It is immediate to see that each term of the sum is the constraint represented by a path between the same pair of nodes. Therefore every relation $D_{1 j}$ in $D$ represents the global contraint transmitted by some set of paths between vertices $V_{i}$ and $V_{j}$. Especially interesting then are the limit relations $\mathrm{D}_{i j}^{*}$ * representing the global constraint transmitted by 11 the paths in $R$ between $V_{i}$ and $V_{j} \cdot D^{*}$ is the corrisponding network, called limit network. The next theorem proves some interesting properties of $D^{*}$ Theorem 5.3 Let $R$ be a star-distributive notwork, let $D^{*}$ be its limit network, let $y^{n}$ be the network obtained after one 1teration of algorithm $c$, and let $Y$ be the closure of $R$. We have:

a) $D^{*}=\cdot Y^{n}$

b) $D^{*}=Y$

Therefore $Y=Y^{n}$ and one iteration is sufficient for algorithm $C$.

* As usual, we assume $D_{i i}^{*} \subseteq I_{i 1}(1=1, \ldots, n)$ 
Breof a) According to theorem (4.4), if $Y_{i j, r s}^{n}=1$, then the pair $\left(x_{i, r}, x_{j, s}\right)$ is allowed by all the paths in $R$. Therefore $Y_{i j}^{n} \subseteq D_{i j}^{*}$

On the other hand, $\mathrm{Y}_{i j}^{\mathrm{n}}$ is obtained, in algorithm $\mathrm{C}$, with a finite number of intersections and compositions of relations of $R$. Therefore $Y_{i j}^{n}$ is the sum of some of the terms of which $D_{i j}^{*}$ is the sum: thus

$$
D_{i j}^{*} \subseteq Y_{i j}^{n}
$$

Therefore we have

$$
D_{i j}^{*}=Y_{i j}^{n} \quad(i, j=1, \ldots, n)
$$

b) It is very easy to see that $D^{*}$ is the solution of system (4.1). We prove first that $D^{*}$ satisfies equations (4.1). In fact, let us consider the relation $D_{i j}^{*}$. It is equal to the sum of the terms corresponding to all paths between $v_{i}$ and $v_{j}$. The first factor of each term must be one of the relations $R_{i k}(k=1, \ldots, n)$. Partitioning the paths and factorizing $R_{i k}$, we clearly obtain the right member

$$
\sum_{k=1}^{n} R_{i k} D_{k j}^{*}+d_{i j}
$$

In fact if $i=j$ the condition $D_{i i}^{*} \subseteq I_{11}$ holds by construction. Since $D^{*}$ satisfies (4.1), from thearem (4.2) we have

$$
D^{*} \subseteq Y
$$

But we have

$$
Y \subseteq Y^{n}
$$

and, from part a)

$$
D^{*}=Y^{n}
$$

Thus we have also

$$
D^{*}=Y
$$


It may be interesting to see how in the star-distributive case algorithm $C$ is nothing else that the solution by Gaussian elimination of the system of equati ons (4.1). We will show it with an example. If $n=3$, wo have

$$
\begin{aligned}
& Y_{1 j}=R_{11} Y_{1 j}+R_{12} Y_{2 j}+R_{13} Y_{3 j}+d_{1 j} \\
& Y_{2 j}=R_{21} Y_{1 j}+R_{22} Y_{2 j}+R_{23} Y_{3 j}+d_{2 j} \quad j=1, \ldots, n \\
& Y_{3 j}=R_{31} Y_{1 j}+R_{32} Y_{2 j}+R_{33} Y_{3 j}+d_{3 j}
\end{aligned}
$$

Now it is easy to see from a truth table that the solution of the single equation

$$
Z=A Z+B
$$

is

$$
Z=A B
$$

if we are interested (as we are) only in the largest $z$. Thus applying distributivity, the first equation of our system becomes:

$$
Y_{1 j}=R_{11} d_{1 j}+R_{11} R_{12} Y_{2 j}+R_{11} R_{13} Y_{3 j}
$$

Then substituting in the other two equations, multiplying and factorizing, we get

$$
\begin{aligned}
& Y_{1 j}=R_{11} d_{1 j}+\quad R_{11} R_{12} Y_{2 j}+\quad R_{11} R_{13} Y_{3 j} \\
& Y_{2 j}=R_{21} R_{11} d_{1 j}+\left(R_{22}+R_{21} R_{11} R_{12}\right) Y_{2 j}+\left(R_{23}+R_{21} R_{11} R_{13}\right) Y_{3 j}+d_{2 j} \\
& Y_{3 j}=R_{31} R_{11} d_{1 j}+\left(R_{32}+R_{31} R_{11} R_{12}\right) Y_{2 j}+\left(R_{33}+R_{31} R_{11} R_{13}\right) Y_{31}+d_{31}
\end{aligned}
$$

The matrix of coefficients of this now system is exactly equal to $Y^{l}$ if we notice that $R_{11} \subseteq I_{11}$ and thus $R_{11}=R_{11} R_{11}$ and furthermore $R_{k I} R_{11}=R_{k l}+R_{k l} R_{1 l}$. After elimination of $Y_{2 j}$ and $Y_{3 j}$ we obtain:

$$
\begin{aligned}
& Y_{1 j}=Y_{11}^{3} d_{1 j}+Y_{12}^{3} d_{2 j}+Y_{13}^{3} d_{3 j} \\
& Y_{2 j}=Y_{21}^{3} d_{I j}+Y_{22}^{3} d_{2 j}+Y_{23}^{3} d_{3 j} \\
& Y_{3 j}=Y_{31}^{3} d_{1 j}+Y_{32}^{3} d_{3 j}+Y_{33}^{3} d_{3 j}
\end{aligned}
$$

For example, if we write the first equation for $j=2$, we have:

$$
Y_{12}=Y_{11}^{3} U_{12}+Y_{12}^{3}+Y_{13}^{3} U_{32}
$$


But it could be possible to see * that

$$
\mathrm{Y}_{i j}^{3} \subseteq \mathrm{Y}_{\mathrm{ik}}^{3} \mathrm{U}_{\mathrm{kj}}
$$

In conclusion we have

$$
Y_{i j}=Y_{i j}^{3}
$$

We can also point out that algorithm $C$ is similar to the Warshall algorithm [1]] for finding the closure of a relation or to the Floyd algorithm [12] for determining, the shortest path between all pairs of nodes in a weighted graph, or to the algorithm for deriving a regular expression from a left linear gramar or from a transition graph [13]. The simi]arity is not casual. In fact all these algorithm can be considered the solution by Gaussian elimination of a linear system of equations in a suitable algebra. We can find the same analogy in Jordan algorithm for matrix inversion in the usual linear algebra. The only difference is that in this case the solution of the single equation

$$
Z=A Z+B
$$

is.

$$
Z=(I-A)^{-1} B
$$

while in our algebra, as already pointed out, the solution is

$$
Z=A B
$$

In what follows we impose restrictions on our relations for obtaining distributive and star-distributive networks.

Let us define a partial ordering $\leq$ in the set $X_{i}$ of values of the variable $x_{i} \cdot^{* *}$ If $x_{1}$ is finite, a complete lattice structure is thus

* Actually, the terms of the type $\mathrm{Y}_{i k}^{3} \mathrm{U}_{\mathrm{kj}}$ would not even exist, if variable elimination had taken place separately for the different values of index $j$. ** For notational simplicity we will consider the partial ordering as defined on the set of indexes as well. For instance $r \leq s$ is equivalent to $x_{i, r} \leq x_{i, s^{*}}$ 
superimposed on $X_{i}$ together with the operations of sup and inf. A total relation $R_{i j}$ between a set $X_{i}$ and a set $X_{j}$ will be called monotone if it has the following properties:

i) (5.2) if $R_{i j, r s}=1$ and $t \geq r$ then $R_{i j, t s}=1$ and conversely if $R_{i j, r s}=1$ and $t \leq s$ then $R_{i j, r t}=1$

ii) (5.3) if $R_{i j, p s}=1, R_{1 j, q s}=1$ and $r=\operatorname{lnf}(p, q)$ then $R_{i j, r s} \neq 1$ and if $R_{i j, r p}=1, R_{i j, r q}=1$ and $s=\sup (p, q)$ then $R_{i j, r s}=1$

The next theorem will clarify the kind of relations allowed by the above definition.

Theorem 5e4 Given a total relation $R_{i j}$, a necessary and sufficient condition for $R$ to be monotone is that a defining function

$$
f_{i j}: \quad x_{i} \rightarrow x_{j}
$$

exists, such that

(5.4) $R_{i j, r s}=1$ iff $s \leq f_{i j}(r)$

and

(5.5) $f_{i j}\left(\inf \left(r_{1}, r_{2}\right)\right)=\inf \left(f_{i j}\left(r_{1}\right), f_{i j}\left(r_{2}\right)\right)$

sr, by duality, that an inverse defining function

$$
g_{i j}: \quad x_{j}-x_{i}
$$

exists, such that

(5.6) $R_{i j, r s}=I$ iff $r \geq g_{i j}(s)$

and

(5.7) $g_{i j}\left(\sup \left(s_{1}, s_{2}\right)\right)=\sup \left(g_{i j}\left(s_{1}\right), g_{i j}\left(s_{2}\right)\right)$

proef We will prove this theorem only for conditions (5.4) and (5.5). The proof in term of the inverse defining function is exactly dual.

Sufficiency. From equation $(5.5)$ we have

$(5.3)$ if $r_{1} \leq r_{2}$ then $f_{i j}\left(r_{1}\right) \leq r_{i j}\left(r_{2}\right)$ 
Therefore if $R_{i j, r s}=1$ and $t \geq r$ from $(5.4)$ and (5.8) we have

$$
s \leq f_{i j}(r) \leq f_{i j}(t)
$$

and thus

$$
R_{i j, t s}=1
$$

If $R_{i j, r s}=1$ and $t \leq s$ we have

$$
t \leq s \leq f_{i j}(r)
$$

and thus

$$
R_{i j, r t}=1
$$

If $R_{i j, r p}=1, R_{i j, r q}=1$ and $s=\sup (p, q)$ we have

$$
p \leq f_{i j}(r) \text { and } q \leq f_{i j}(r)
$$

and thus

$$
s=\sup (p, q) \leq f_{i j}(r)
$$

therefore

$$
\begin{aligned}
& R_{i j, r s}=1 \\
& \text { If } R_{i j, p s}=I, R_{i j, q s}=1 \text { and } r=\inf (p, q) \text { we have } \\
& s \leq f_{i j}(p) \quad \text { and } s \leq f_{i j}(q)
\end{aligned}
$$

and thus

$$
s \leq \inf \left(f_{i j}(p), f_{i j}(q)\right)=f_{i j}(r)
$$

therefore

$$
R_{i j, r s}=1
$$

Necessity If a relation $R_{i j}$ is monotone $i t$ can be put in the form (5.4). In fact, given an element $r$ of $x_{i}$ let us compute the superior $f_{i j}(r)$ of the image of $r$ in $R_{i j^{\bullet}}$ for (5.3) we have

$$
R_{i j, r f_{i j}}(r)=I
$$

Thus, for (5.2), equation (5.4) is satisfied. Function $f_{i f}(r)$ satisfies equation (5.5). In fact, for (5.2) we have: 


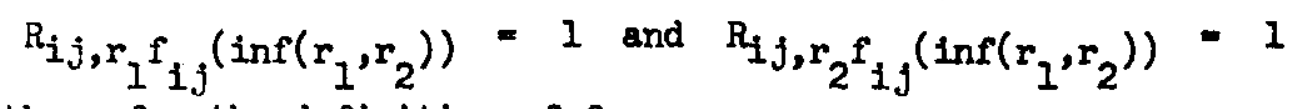

and thus, for the definition of $f$ :

$$
f_{i j}\left(\inf \left(r_{1}, r_{2}\right)\right) \leq f_{i j}\left(r_{1}\right) \text { and } f_{i j}\left(\inf \left(r_{1}, r_{2}\right)\right) \leq f_{i j}\left(r_{2}\right)
$$

Therefore

$$
\text { (5.9) } f_{i j}\left(\inf \left(r_{1}, r_{2}\right)\right) \leq \inf \left(f_{i j}\left(r_{1}\right), f_{i j}\left(r_{2}\right)\right)
$$

On the other hand, we have

From (5.2)

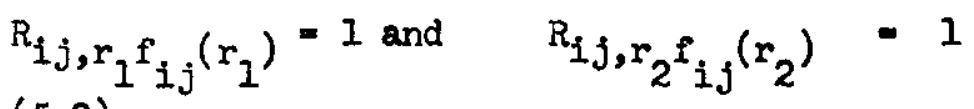

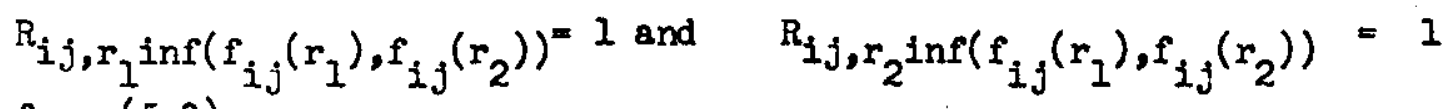

Thus from (5.3)

Therefore

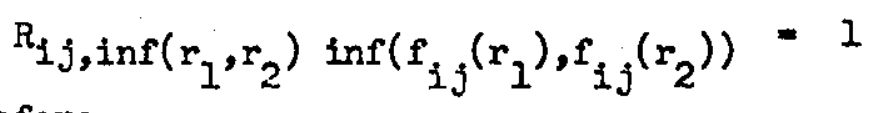

$$
f_{i j}\left(\inf \left(r_{1}, r_{2}\right) \geq \operatorname{lnf}\left(f_{i j}\left(r_{1}\right), f_{i j}\left(r_{2}\right)\right)\right.
$$

Finally from the above relation and (5.9), equation (5.5) follows.

$$
\text { Q.E.D. }
$$

A few examples will clarify the kind of relations allowed by the monotonicity constraint. For instance, if the partial ordering is also total, equation (5.5) can be substituted by equation (5.8), i.e. the defining function must be monotone. In Fig. 6a we see a monotone relation represented by a bipartite graph. We have $f_{i j}(5)=4, f_{i j}(4)=f_{i j}(3)=2, f_{i j}(2)=f_{i j}(1)=1$. Conversely, $g_{i j}(1)=1, g_{i j}(2)=3, g_{i j}(3)=g_{i j}(4)=5$. A special case of monotone relation, with infinite sets, is represented by the "shortest path" constraint

$$
s \leq f_{i j}(r)=r+d
$$

In fact, the shortest path problem in a weighted graph is a special case of our central problem. The network of relations $R$ can be obtained from the 
weighted graph as follows. The set of values for each variable is the set of integers and all relations $R_{i j}(i, j=1, \ldots, n)$ are monotone. If $R_{i j}$ is specified by the defining function $f_{i j}^{R}$ we have

$$
x_{j} \leq f_{i j}^{R}\left(x_{i}\right)=x_{i}+t_{i j}
$$

where $t_{i j}$ are the arcs weights : $t_{i j}=t_{j i}, t_{i i}=0$

We will see that the minimal network $M$ has the same form:

$$
x_{j} \leq f_{i j}^{M}\left(x_{i}\right)=x_{i}+d_{i j}
$$

and thus $d_{i j}$ represents the length of the shortest path from vertex $v_{i}$ to vertex $\nabla_{j} \cdot$ As a check, note that $d_{i j} \leq t_{i j}$ and so $M_{i j} \subseteq R_{i j}$.

If the sets $X_{i}$ are finite, the restriction to total relations could look heavy. On the contrary, a"floor" value $x_{i, 0}$ and a "ceiling" value $x_{i, u}$ can always be added to $\mathrm{X}_{i}$ such that

$$
R_{i, j, O_{s}}=I \text { for all s and } R_{j i, r u}=I \text { for all } r
$$

Fig. 5b shows the relation in Fig.5a without ceiling in $X_{1}$ and floor in $X_{j}$. In general, monotone relations are considerably more powerful than simple "shortest path" constraints. For instance, the lattice structure of multidimensional euclidean spaces can be used for specifying multidimensional rectangular domains. In Fig $6 \mathrm{c}$ we see how defining functions $f_{i j}$ of $R_{i j}$ and $g_{j i}$ of $R_{j i}$ restrict to a rectangle the image of $x_{i, r}$ in $R_{i j}+R_{j i}$. Different points $x_{i, r}$ can generate different rectangles, provided equation (5.5) is satisfied.

The next theorem proves the closure of the class of monotone relations under the operations of intersection and composition and gives the rules for performing such operations in terms of the defining functions. Theorem 5.5 a) If $R_{i j}^{\prime}$ and $R_{i j}^{\prime \prime}$ are monotone relations represented by the defining functions $f_{i j}^{\prime}$ and $f_{i j}^{\prime \prime}$, then the sum: 


$$
R_{i j}=R_{i j}^{\prime}+R_{i j}^{\prime \prime}
$$

is a monotone relation representod by the defining function

(5.10) $f_{i j}(r)=\operatorname{lnf}\left(f_{i j}^{i}(r), f_{i j}^{\prime \prime}(r)\right)$

b) Likewise, the product

$$
R_{i j}=R_{i k} R_{k j}
$$

is represented by

$$
\text { (5.II) } f_{i j}(r)=f_{k j}\left(f_{i k}(r)\right)
$$

Proof a) iRelation $R_{i j}$ defined by $(5.4)$ and $(5.10)$ is evidently the intersection of $R_{i j}^{\prime}$ and $R_{i j}^{\prime \prime}$. Furthermore $R_{i j}$ is total. In fact we have at least

$$
R_{i j, r f_{i j}}(r)=1 \text { and } \quad R_{i j, g_{j j}}(s) s=1
$$

where $g(s)$ is defined dually. Finally, function $f_{1 j}$ satisfies equation (5.5):

$$
\begin{aligned}
f_{i j}\left(\inf \left(r_{1}, r_{2}\right)\right) & =\inf \left(f_{i j}^{\prime}\left(\inf \left(r_{1}, r_{2}\right)\right), f_{i j}^{\prime \prime}\left(\inf \left(r_{1}, r_{2}\right)\right)\right)= \\
& =\inf \left(\inf \left(f_{i j}^{\prime}\left(r_{1}\right), f_{i j}^{\prime}\left(r_{2}\right)\right), \inf \left(f_{i j}^{\prime \prime}\left(r_{1}\right), f_{i j}^{\prime \prime}\left(r_{2}\right)\right)\right)= \\
& =\inf \left(\inf \left(f_{i j}^{\prime}\left(r_{1}\right), f_{i j}^{\prime \prime}\left(r_{1}\right)\right), \inf \left(f_{i j}^{\prime}\left(r_{2}\right), f_{i j}^{\prime \prime}\left(r_{2}\right)\right)\right)= \\
& =\inf \left(f_{i j}\left(r_{1}\right), f_{i j}\left(r_{2}\right)\right)
\end{aligned}
$$

b) The "if" part of (5.4) is trivial. For the"only if" part, if $R_{1 j, r s}=I$ then an index $t$ exists, such that $R_{i k, r t}=1$ and $R_{k j, t s}=1$. But $R_{i k, r t}=1$ implies $t \leq f_{i k}(r)$. Thus from $(5.8)$ we have

$$
f_{k j}(t) \leq f_{k j}\left(f_{i k}(r)\right)=f_{i j}(r)
$$

But $R_{k j, t s}=1$ implies $s \leq f_{k j}(t)$ and thus $s \leq f_{i j}(r)$. Relation $R_{i j}$ is also total because at least

$$
R_{i j, r_{i j}}(r)=1 \text { and } R_{i j, g_{i j}(s) s}=1
$$

Equation (5.5) is proved as follows:

$$
\begin{aligned}
f_{i j}\left(\inf \left(r_{1}, r_{2}\right)\right) & =f_{k j}\left(f_{i k}\left(\inf \left(r_{1}, r_{2}\right)\right)=f_{k j}\left(\inf \left(f_{i k}\left(r_{1}\right), f_{i k}\left(r_{2}\right)\right)\right)=\right. \\
& =\inf \left(f_{k j}\left(f_{i k}\left(r_{1}\right)\right), f_{k j}\left(f_{i j}\left(r_{2}\right)\right)\right)=\inf \left(f_{i j}\left(r_{1}\right), f_{i j}\left(r_{2}\right)\right)
\end{aligned}
$$


Next theorem proves the distributivity of monotone relations.

Theorem $5.6 \quad$ a) Any set of monotione relations

$R_{i k} \quad, \quad R_{k j} \quad i, j=1, \ldots, n$

form a distributive set of relations with respect to set $\mathrm{X}_{k}$, i.e.

(5.12) $\left(\sum_{i=1}^{p} v_{0 i} R_{i k}\right)\left(\sum_{j=1}^{q} R_{k j} v_{j 0}\right)=\sum_{j=1}^{p} \cdot \sum_{j=1}^{q} v_{0 i} R_{i k} R_{k j} v_{j 0}$

for all fundamental vectors $\mathrm{V}_{\mathrm{O}}$ and $\mathrm{V}_{j 0^{\circ}}$

Proof For theorem(5.4) vectors $R_{O k}^{i}=V_{O i} R_{i k}$ and $R_{k 0}^{j}=R_{k j} V_{j 0}$ represent subsets of $X_{k}$ of the form

(5.13) $R_{0 k, t}=1$ iff $t \leq \bar{t}$ and $R_{k 0, t}=1$ iff $t \geq \bar{t}$

Furtiermore, intersection of two subsets of this form produces a subset of

the same form. If

$$
\mathrm{R}_{\mathrm{OK}}=\mathrm{R}_{\mathrm{OK}}^{\prime}+\mathrm{R}_{\mathrm{OK}}^{\prime \prime}
$$

we have

$$
\mathrm{R}_{\mathrm{O}, \mathrm{t}}=1 \text {. iff } \quad t \leq \inf \left(\mathrm{t}^{-1}, \mathrm{t}^{-1 t}\right)
$$

And if

$$
R_{k 0}=R_{k 0}^{\prime}+R_{k 0}^{\prime \prime}
$$

we have

$$
r_{k 0, t}=1 \text { iff } \quad t \geq \sup \left(\vec{t}^{\prime}, t^{-1}\right)
$$

We will prove that subsets of this form satisfy both left and right distributivity:

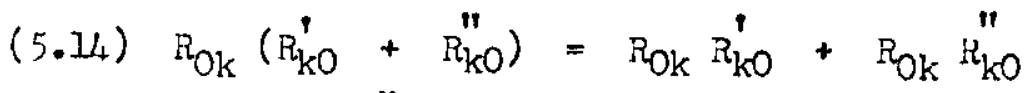

(5.15) $\left(R_{O k}^{\prime}+R_{O k}^{\prime \prime}\right) R_{k O}=R_{O K}^{\prime} R_{k O}+R_{O k}^{n} R_{k O}$

In fact, the right member of (5.1), in binary form, is:

$$
\left(\bigvee_{t=1}^{N} R_{0 k, t} \Lambda R_{k 0, t}^{\prime}\right) \Lambda\left(\bigvee_{t=1}^{N} R_{0 k, t} \Lambda R_{k 0, t}^{\prime \prime}\right)
$$

Applying binary distributivity, we have

$$
\int_{t_{1}=1}^{N} V_{t_{2}=1}^{N}\left(R_{0 k, t_{2}}^{N} \Lambda \cdot R_{0 k, t_{2}}\right) \Lambda\left(R_{k 0, t_{1}}^{\prime} \Lambda R_{k 0, t_{2}}^{\prime \prime}\right)
$$


or equivalently

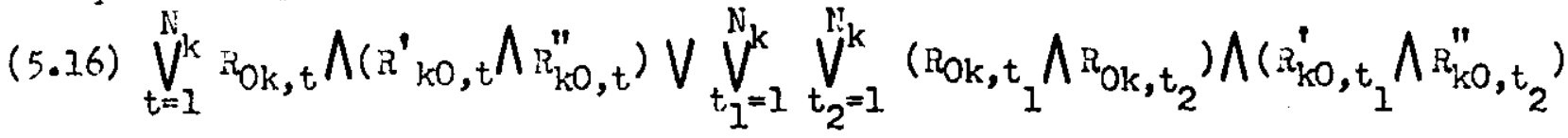

From (5.13), if

tren a value

$$
\mathrm{R}_{\mathrm{Ok}, \mathrm{t}_{1}}=1, \mathrm{R}_{\mathrm{ok}, \mathrm{t}_{2}}=1, \mathrm{R}_{\mathrm{kO}, \mathrm{t}_{1}}^{\prime}=1 \text { and } \mathrm{R}_{\mathrm{k} 0, \mathrm{t}_{2}}^{\prime \prime}=1
$$

$$
t=\sup \left(t_{1}, t_{2}\right)
$$

can be found, such that

$$
R_{\mathrm{Ok}, t}=1, \quad \mathrm{R}_{\mathrm{kO}, \mathrm{t}}^{\prime}=1 \text { and } \mathrm{R}_{\mathrm{kO}, \mathrm{t}}^{\prime \prime}=1
$$

Therefore equation $(5.16)$ becomes

$$
\bigvee_{t=1}^{N} R_{0 k, t} \Lambda\left(R_{k 0, t}^{\prime} \Lambda R_{k 0, t}^{\prime \prime}\right)
$$

i.e., the left member of $\left(5.1 u_{4}\right)$. Formula (5.15) can be proved dually.

From closure under sum, left and right distributivity we have:

$\left(\sum_{i=1}^{p} R_{0 k}^{i}\right)\left(\sum_{j=1}^{q} R_{k 0}^{j}\right)=\sum_{i=1}^{p} \sum_{j=1}^{q} R_{0 k}^{i} R_{k 0}^{j}$

i.e., formula $(5.12)$

Q.E.D.

The next corollary will be useful in establishing star-distributivity. Corellary If $R_{i k}, R_{i k}^{\prime}, R_{i k}^{\prime \prime}, R_{k j}, R_{k j}^{\prime}$ and $R_{k j}^{\prime \prime}$ are monotone relations we have:

$$
\begin{aligned}
& (5.17) R_{i k}\left(R_{k j}^{\prime}+R_{k j}^{\prime \prime}\right)=R_{i k} R_{k j}^{\prime}+R_{i k} R_{k j}^{\prime \prime} \\
& (5.18)\left(R_{i k}^{\prime}+R_{i k}^{\prime \prime}\right) R_{k j}=R_{i k}^{\prime} R_{k j}+R_{i k}^{\prime \prime} R_{k j}
\end{aligned}
$$

Proof. Equation (5.17) can be written as

$$
v_{0 i} R_{i k}\left(R_{k j}^{\prime} v_{j O}+R_{k j}^{\prime \prime} v_{j 0}\right)=v_{0 i} R_{i k} R_{k j}^{\prime} v_{j O}+v_{0 i} R_{i k} R_{k j}^{\prime \prime} v_{j O}
$$

for all fundamental vectors $\mathrm{V}_{\mathrm{O}_{i}}$ and $\mathrm{V}_{j 0^{\circ}}$. Therefore it descends from (5.12). The same is true for $(5.18)$. 
We can now prove our final result.

Theorem 5.7 Let $R$ be a network of relations such that:

i) its relations $R_{i j}(i, j=1, \ldots, n ; i \neq j)$ are monotone and

ii) the loop relations $Y_{i j}^{n}(i=I, \ldots, n)$ of the network $Y^{n}$ obtained after one j.teration of algorithm $C$ are equal to unity.

We can prove that:

a) Network $R$ is star-distributive.

b) Network $Y^{n}$ is equal to the closure $Y$ of $R$. All relations

$Y_{i j}\left(i, j=1, \ldots, n ; i_{j}^{l} j\right)$ are monotone.

c) Network $Y$ is distributj.ve.

d) Network $R$ is decomposable and the symmetrization $Y^{\prime}$ of its closure $Y$ is minimal.

Proof a) From condition $i i)$ and $Y^{n} \subseteq R$ we have $R_{i i}=I_{i i}$. Expressions obtained by combining relations $R_{i j}(i, j=1, \ldots, n ; 1 \neq j)$ with the operations of intersection and composition evaluate to monotone relations for theorem (5.5). The unity elements $R_{i i}$ can be involved in an expression either under composition or under intersection. In the former case a monotone relation is trivially obtained. In the latter case the unity $I_{i i}$ must be intersected with an expression representing the global constraint given by a set of circuits from $V_{i}$ to $V_{i}$ in $R$. In fact we can assume inductively that no unity is involved in this expression, and in this case distributivity holds for the corollary to theorem (5.6), and the expression can always be reduced to a sum of products. The result of the intersection operation must be again unity, because $Y_{i i, r r}^{n}=I$ for all $r$, and thus for theorem $(4.4)$ all pairs $\left(x_{i, r}, x_{i, r}\right)$ must be allowed by all circuits from $V_{i}$ to $V_{i}$ in $R$. In conclusion, the set $D$ of all expressions contains expressions that ejther evaluate to monotone relations or to identities. 
Left distributivity

(5.19) $E_{i k}\left(E_{k j}^{\prime}+E_{k j}^{\prime \prime}\right)=E_{i k} E_{k j}^{\prime}+E_{i k} E_{k j}^{\prime \prime}$

always hold. In fact, if all relations are monotone, this property is proved by the corollary to theoren $(5.6)$. If $\mathrm{E}_{i \mathrm{k}}$ is a unity, both members evaluate to $E_{k j}^{\prime}+E_{k j}^{\prime \prime}$. If $E_{k j}^{p}$ or $E_{k j}^{\prime \prime}$ is a unity, say $E_{k j}^{\prime}$, we have $k=j$ and (5.19) becomes $E_{i k}\left(I_{k k}+E_{k k}^{\prime \prime}\right)=E_{i k} I_{k k}+E_{i k} E_{k k}^{\prime \prime}$

But then $E_{k k}=I_{k k}$ for $Y_{k k}^{n}=I_{k k}$, and therefore both members evaluate to $E_{i k}$. The same proof holds for right distributivity.

b) This part follows from a) and theorem (5.3).

c) This part follows from $b$ ) and theorem (5.6).

d) This part follows from c) and theorem (5.2). 


$$
=45=
$$

\section{Cenclusion}

In this paper we have presented a formal treatenent of networks of binary constraints. The main practical result was the discovery of an algorithm for adding to the direct constraint between each pair of variables the indirect constraints transmitted by all the paths in the network. In particular cases the resulting constraint was proved equivalent to the global constraint represented by the entire network as seen by that pair of vertices. This result allows the partial or total utilization of the global constraint structure for reducing the set of feasible values of a variable to be determined, when the values of other variables are known.

For the practical computer implementation of this method, the following requirements can be surgested:

a) In the application under examination, most constraints must be reasonably represented or approximated by binary constraints or simple networks of binary constraints. Note that if we allow a constraint among $m$ variables to be represented by a network of $n$ vertices, with $n>m$, then the negative result of section 3 no longer holds, and many representations of the constraint, trivial and not, can be found. For instance, the ternary relation (3.4) which is not representable with a 3-vertex network, can be represented by the 4 -vertex network in Fig. 2, as seen from vertices $V_{1}, V_{2}$ and $V_{3}$. b) The resulting binary relations (finite or infinite) must be capable of being stored in an economical way in a computer memory. For instance, if the variables are points of m-dimensional spaces, a relation $R_{i j}$ could be stored representing the images in $x_{j}$ of all elements ${ }^{*} x_{i, r}$ of $x_{i}$ as m-dimensional

* Or just one, if all the other images can be obtained from it by a fixed procedure (e.g. translation). 


$$
=146=
$$

domains. Known tecniques of domain encoding can then be used. For instance, two given points are sufficient for determining a rectangular domain: this is often the meaning of functions $f_{i j}(r)$ and $g_{j i}(r)$ representing a monotone relation.

c) The operations of intersection and composition must be easily definable in the chosen class of relations. In particular, this class must be closed under those two operations. For instance, this is the case of relations represented by domains, convex domains, domains enclosed by polygons or convex polygons, rectangular domains.

d) The closed network is then obtained with algorithm $C$. The closed network should then be close to the minimal. For instance, we have coincidence for rectangular domains, and we expect reasonable closeness for convex domains. Bad results can be expected if the relations allow most pairs and forbide a few isolated pairs, like in grapi. coloring problems. Anyway, if the addition of a further constraint destroys regularity (i.e. closed $\neq$ minimal), it is, nevertheless, convenient to add 1t. Maybe its addition will not be entirely exploited, but the monotonicity property of intersection and composition certifies that the modified closed network will be more restrictive. 


\section{References}

[1] Eastman, C. M., Representations for space planning, CACM 13, 4, (April 1970) pp $242-250$.

[2] Guzman, A., Computer recognition of three-dimensional objects in a visual scene, MAC-TR-59 Project MAC Massachusetts Institute of Technology, December 1968, PhD thesis.

[3] Kelly, M.D., Edge detection in pictures by computer using planning, AIN=108 Stanford Artificial Intelligence Project, Stanford University, January 1970.

[4] Montanari, U.G., On the optimal detection of curves in noisy pictures, AIM-115 Stanford Artificial Intelligence Project, Stanford University, also CACM, in press.

[5] Narasimhan, R., On the description, generation and recogrittion of classes of pictures, in A.Grasselli Ed, Automatic interpretation and classification of images (Academic Press, 1969).

[6] Clowes, M.B., Transformational g̀rammars and the organization of pictures, in A.Grasselli Ed, Automatic interpretation and classification of imaces (Academic Press, 1969).

[7] Feder, J., Linguistic specification and analysis of classes of line patterns, 4Q3-2 Dept of Electrical Engineering, New York University, April 1969, PhD thesis.

[8] Shaw, A.C., The formal description and parsing of pictures, SLAC_El Stanford Linear Accelerator Center, Stanford University, March 1968 , PhD thesis. 
[9] Anderson, R.li., Syntax-directed recognition of hand-printed two-dimensional mathematics, Applied mathematics, Harvard University, 1968 , PhD. Thesis.

[10] Montanari, U.G., Separable graphs, planar graphs and web grammars, Information and Control 16, 3, (May 1970) pp. 243-267.

[11] Warshall, S., A theorem on boolean matrices, JACM 9, (1962), pp. 11-12.

[12] Floyd, R. W., Algorithm 97 Shortest path, CACM, Vol. 5, 6 (June 1962),345.

[13] Ginzburg, A., Algebraic theory of automata, Academic Press (1968).

[14]: Schwabel, J.C. and McCormick, B., Consistent properties of composite formation under a binary relation, Information Sciences 2 (1970), pp 179-209. 

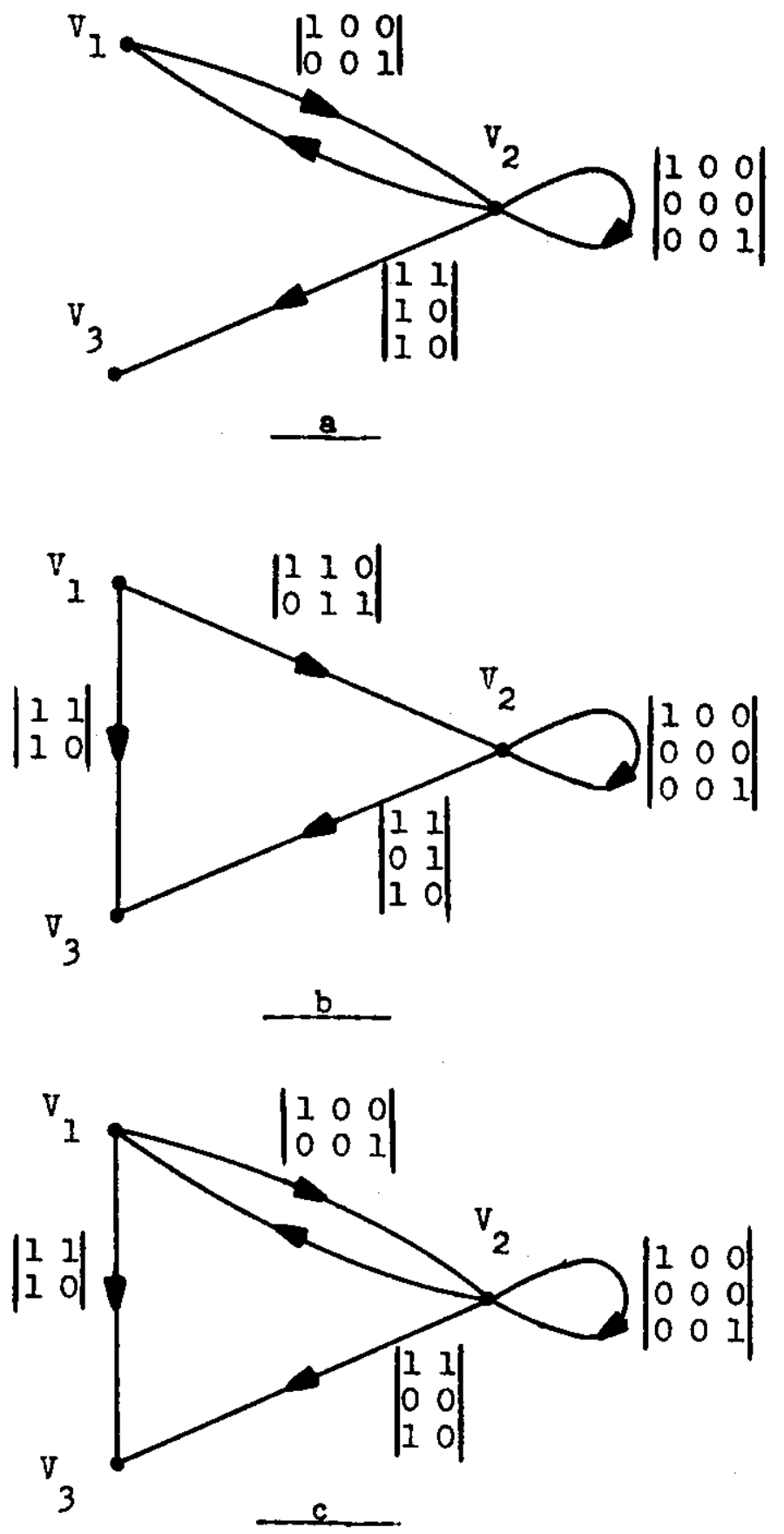

Fij. 1. Examples of networks of constraints. As a graphical convention, if both arcs $V_{j} V_{j}$ and $V_{j} V_{1}$ exist, but only $V_{i} V_{j} i_{s}$ labeled with relation $R_{j j}$ are equivalent, and network c) is the intersection of networks a) and b). 


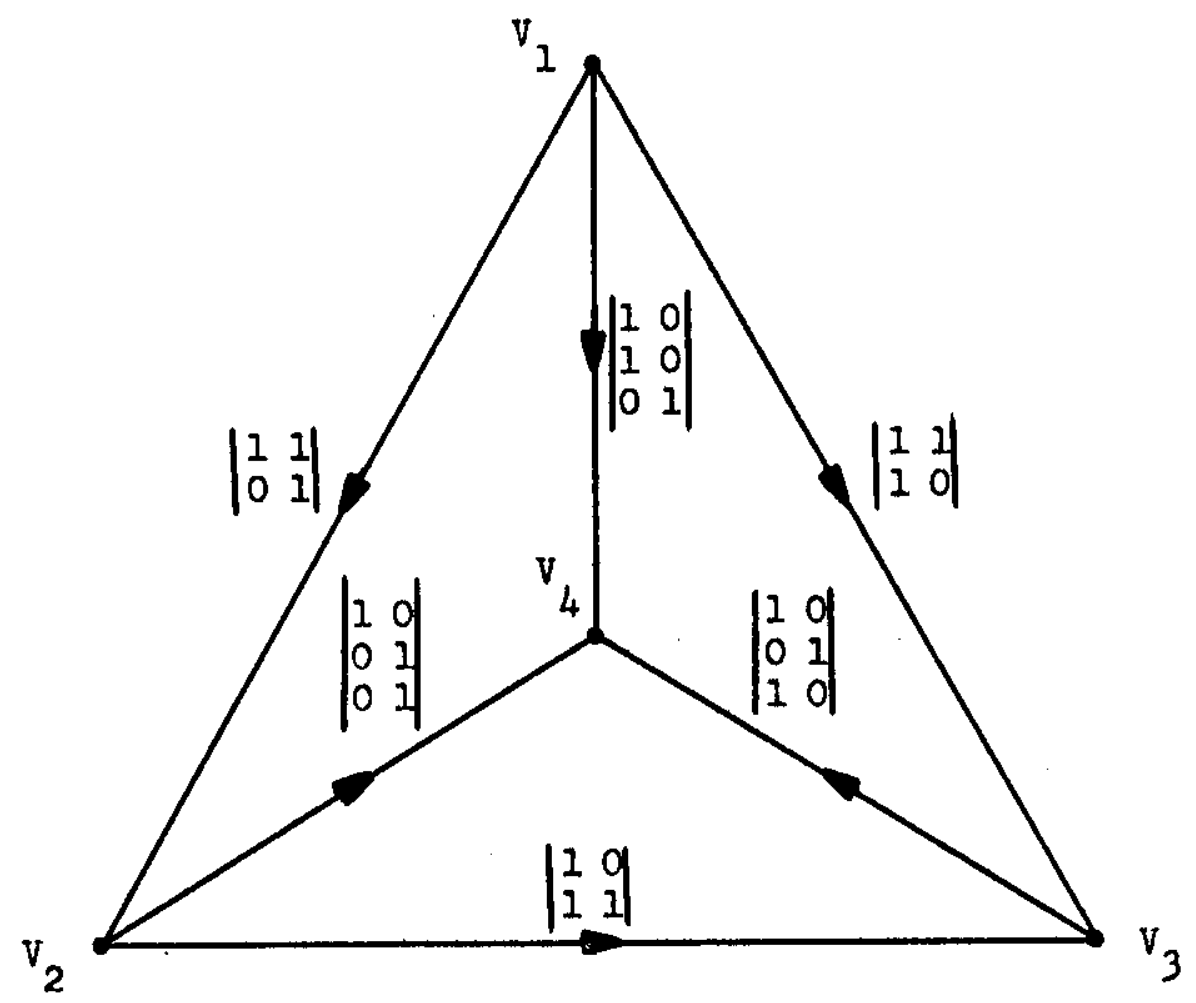

Eife_2. An example of indecomposable network. 


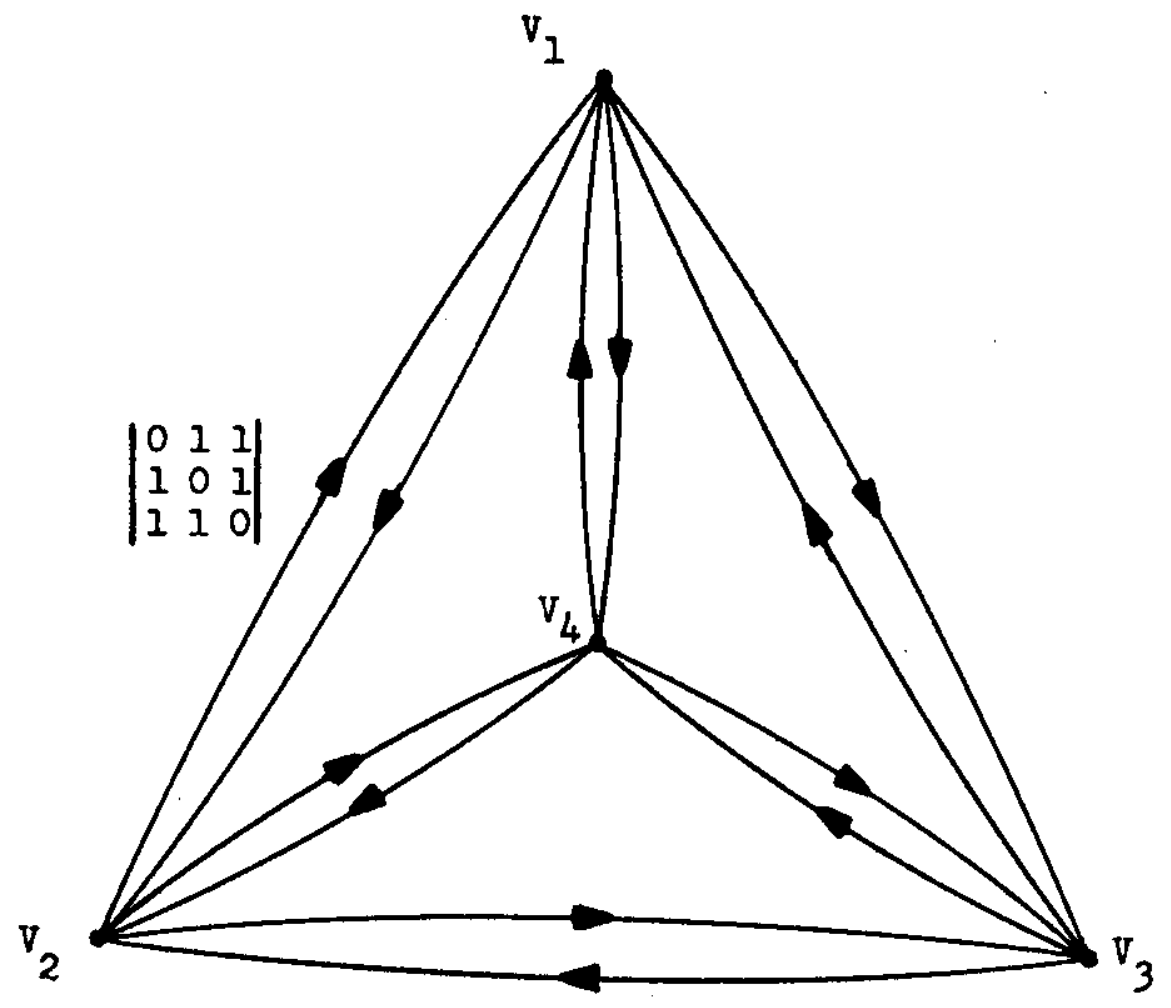

Eige 3. In this network, the relation $\left|\begin{array}{lll}0 & 1 & 1 \\ 1 & 0 & 1 \\ 1 & 1 & 0\end{array}\right|$ is associated to every arc. This network represents the impossible problem of coloring a four-vertex complete graph with three colors. This network is symetric and closed but not minimal. 

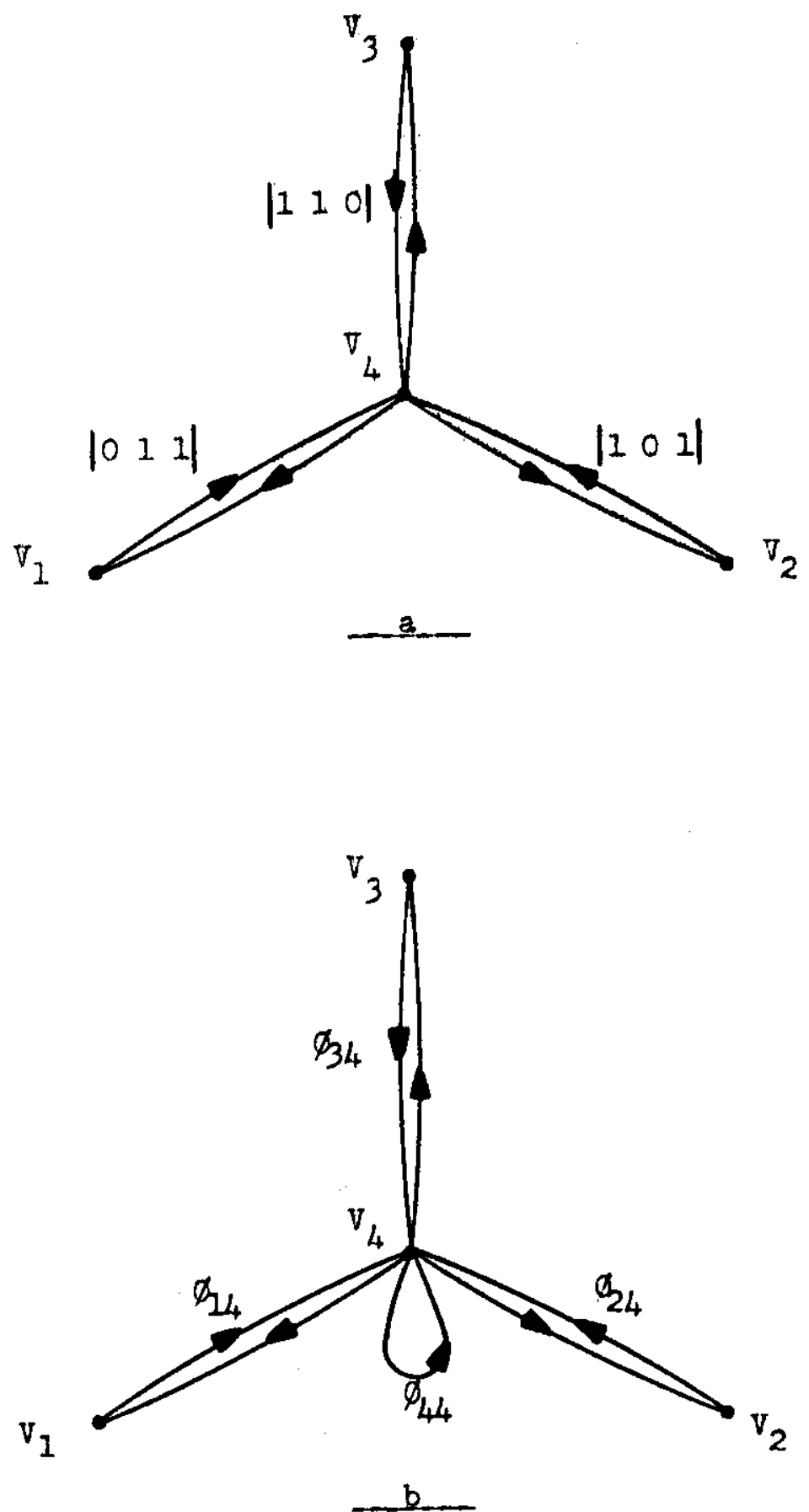

Elgele. a) A symetric notwork of constraints.

b) The network of constraints $Y^{\prime}$ equivalent to a) computed by algorithm $C$ in one iteration, with order of olimination $(1,2,2,3)$. 

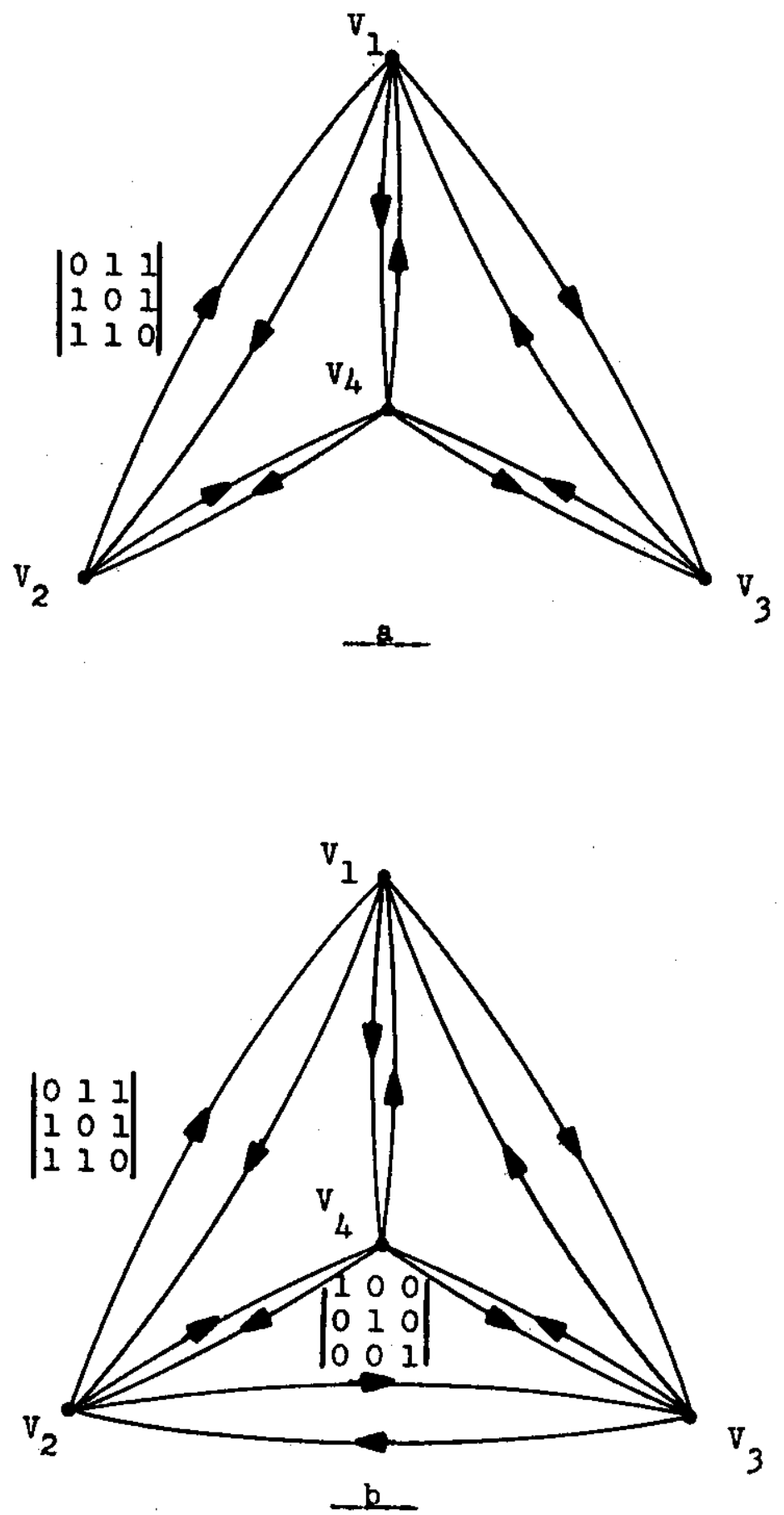

Eige 5e a) A series-parallel network with respect to all pairs of vertices except $\mathrm{V}_{2} \mathrm{~V}_{3}$ b) Its minimal equivalent network. All nonlabelled arcs are assumed labeled with $\left|\begin{array}{lll}0 & 1 & 1 \\ 1 & 0 & 1 \\ 1 & 1 & 0\end{array}\right|$. 

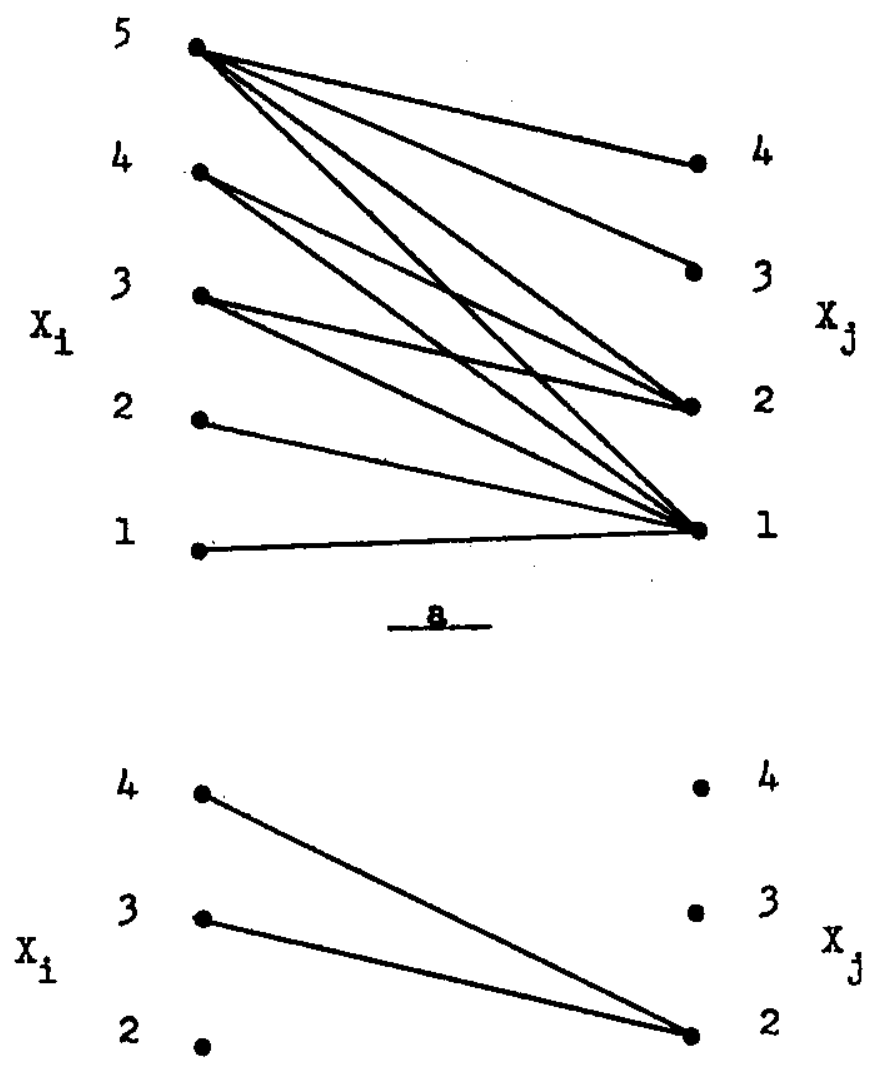

1
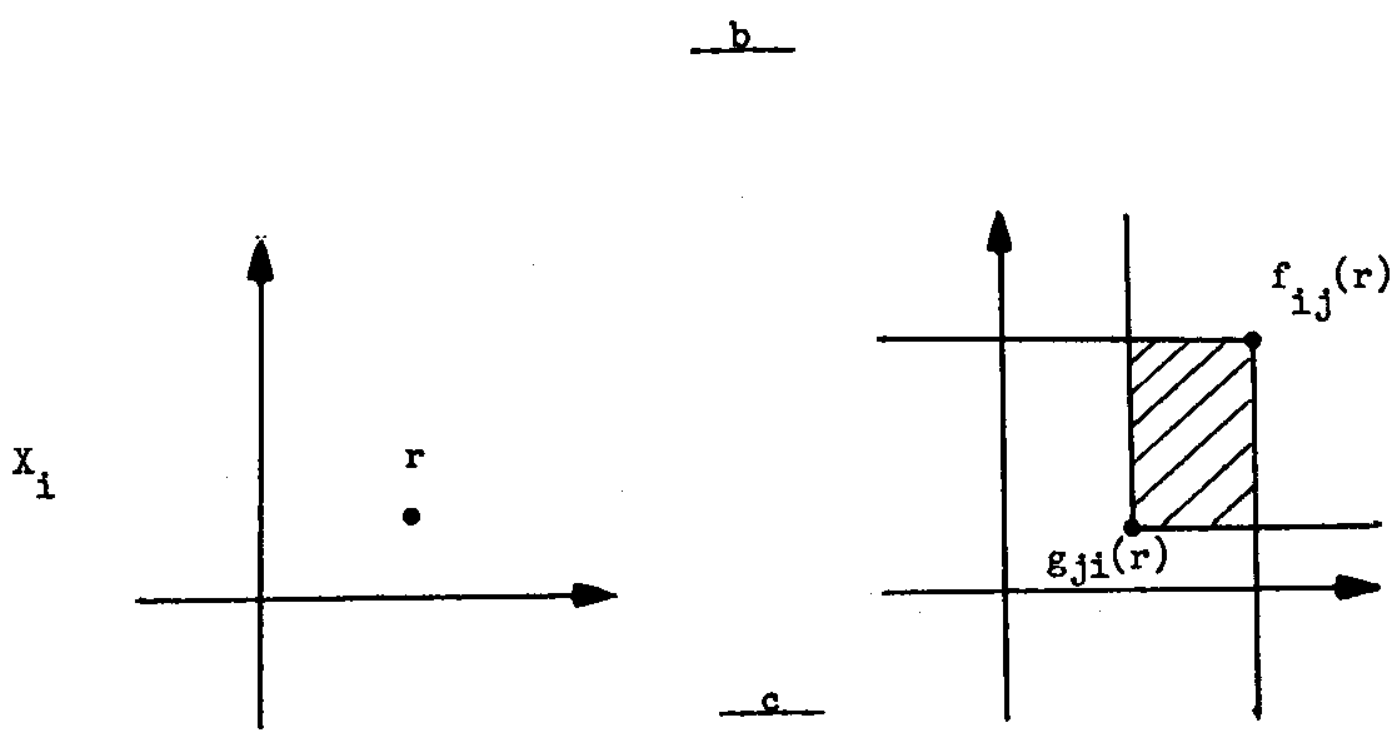

Eige 6. a) An example of monotone relation. without "ceiling" in $\mathrm{X}_{j}$ and "floor" in $\mathrm{X}_{j}$.

b) The relation in a) c) The rectangular image of element $x_{j, r}$ as allowed by the Intersection of two monotone relations $R_{i j}$ and $R_{j i}$. 


\begin{tabular}{|c|c|}
\hline \multicolumn{2}{|c|}{$\begin{array}{c}\text { DOCUMENT CONTROL DATA - R \& D } \\
\text { (Security clas sification of thts, body of atstract and indexing annotation must be ontered when the overall report is classified) }\end{array}$} \\
\hline \multirow{2}{*}{$\begin{array}{l}\text { OAIGINATING ACTIVITY (Corporare author) } \\
\text { Department of Computer Science } \\
\text { Carnegie-Me1lon University } \\
\text { Pittsburgh, Pennsylvania } 15213\end{array}$} & \begin{tabular}{l|l} 
2E. REPORT SECURITY CLASSIFICA TION \\
UNCLASSIFIED
\end{tabular} \\
\hline & 2b. GROUP \\
\hline \multicolumn{2}{|c|}{$\begin{array}{l}\text { Network of constraints: fundamental properties and applications to picture } \\
\text { processing }\end{array}$} \\
\hline \multicolumn{2}{|l|}{$\begin{array}{l}\text { 4. DESCRIPTIVE NOTES (Type ol report and inclusive dates) } \\
\text { Sclentific } \\
\end{array}$} \\
\hline \multicolumn{2}{|l|}{$\begin{array}{l}\text { 5. AUTHORISt (First name, middle initial, last name) } \\
\text { UgO Montanari }\end{array}$} \\
\hline $\begin{array}{l}\text { 6. REPORT OATE } \\
\text { January } 1971\end{array}$ & \begin{tabular}{|l|c} 
78. TOTAL NO. OF PAGES & $\begin{array}{c}\text { D. NO. OF REFS } \\
57\end{array}$ \\
\end{tabular} \\
\hline \multirow{2}{*}{$\begin{array}{l}\text { 8a. CONTRACT OR GRANY NO. } \\
\text { F44620-67-C-0058 } \\
\text { b. PROJECT NO. } \\
\text { A0827-5 } \\
611010 \\
\text { c. }\end{array}$} & Oa. ORIGINATOR'S REPORT NUMEERISI \\
\hline & $\begin{array}{l}\text { 9b. OTHER REPORT NO(S) (Any Other numbers that may be as signed } \\
\text { ihis report) }\end{array}$ \\
\hline \multicolumn{2}{|l|}{ 10. DISTRIBUTION STATEMENT } \\
\hline \multicolumn{2}{|c|}{ 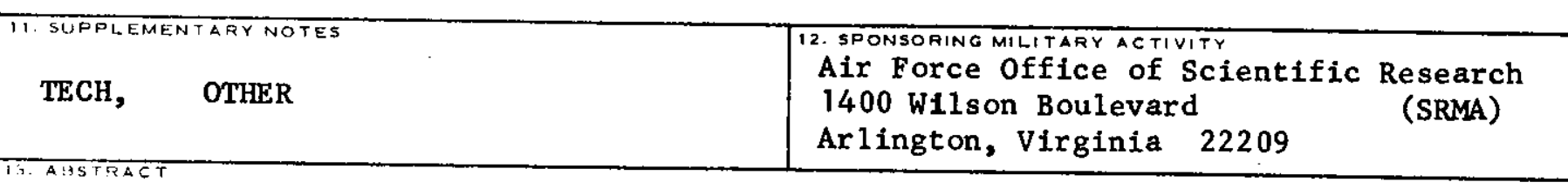 } \\
\hline \multicolumn{2}{|c|}{$\begin{array}{l}\text { The problem of representation and handling of constraints is here considered, } \\
\text { mainly for picture processing purposes. A systematic specification and utilization } \\
\text { of the available constraints could significantly reduce the amount of search in } \\
\text { picture recognition. On the other hand, formally stated constraints can be embedded } \\
\text { in the syntactic productions of picture languages. Only binary constraints are } \\
\text { treated here, but they are represented in full generality as binary relations. Con- } \\
\text { straints among more than two varlables are then represented as networks of simul- } \\
\text { taneous binary relations. In general, more than one equivalent (1.e., representing } \\
\text { the same constraint) network can be found: a minimal equivalent network is shown to } \\
\text { exist, and its computation is shown to solve most practical problems about constraint } \\
\text { handling. No exact solution for this central problem was found. Anyway, constraints } \\
\text { are treated algebraically, and the solution of a system of linear equations in this } \\
\text { algebra provides an approximation of the minimal network. This solution is then } \\
\text { proved exact in special cases, e.g., for tree-like and series parallel networks and } \\
\text { for classes of relations for which a distributive property holds. This latter } \\
\text { condition is satisfied in cases of practical interest. }\end{array}$} \\
\hline
\end{tabular}


